\title{
EXPLORING BIASES OF ATMOSPHERIC RETRIEVALS IN SIMULATED JWST TRANSMISSION SPECTRA
} OF HOT JUPITERS

\author{
M. Rocchetto ${ }^{1}$, I. P. Waldmann ${ }^{1}$, O. Venot $^{2}$, P.-O. Lagage ${ }^{3,4}$, And G. Tinettio ${ }^{1}$ \\ ${ }^{1}$ Department of Physics \& Astronomy, University College London, Gower Street, WC1E6BT London, UK; m.rocchetto@ucl.ac.uk \\ ${ }^{2}$ Instituut voor Sterrenkunde, Katholieke Universiteit Leuven, Celestijnenlaan 200D, B-3001 Leuven, Belgium \\ ${ }^{3}$ Irfu, CEA, Université Paris-Saclay, F-9119 Gif-sur Yvette, France \\ 4 AIM, Université Paris Diderot, F-91191 Gif-sur-Yvette, France \\ Received 2016 August 2; revised 2016 September 26; accepted 2016 September 28; published 2016 December 12
}

\begin{abstract}
With a scheduled launch in 2018 October, the James Webb Space Telescope (JWST) is expected to revolutionize the field of atmospheric characterization of exoplanets. The broad wavelength coverage and high sensitivity of its instruments will allow us to extract far more information from exoplanet spectra than what has been possible with current observations. In this paper, we investigate whether current retrieval methods will still be valid in the era of JWST, exploring common approximations used when retrieving transmission spectra of hot Jupiters. To assess biases, we use 1D photochemical models to simulate typical hot Jupiter cloud-free atmospheres and generate synthetic observations for a range of carbon-to-oxygen ratios. Then, we retrieve these spectra using TauREx, a Bayesian retrieval tool, using two methodologies: one assuming an isothermal atmosphere, and one assuming a parameterized temperature profile. Both methods assume constant-with-altitude abundances. We found that the isothermal approximation biases the retrieved parameters considerably, overestimating the abundances by about one order of magnitude. The retrieved abundances using the parameterized profile are usually within $1 \sigma$ of the true state, and we found the retrieved uncertainties to be generally larger compared to the isothermal approximation. Interestingly, we found that by using the parameterized temperature profile we could place tight constraints on the temperature structure. This opens the possibility of characterizing the temperature profile of the terminator region of hot Jupiters. Lastly, we found that assuming a constant-with-altitude mixing ratio profile is a good approximation for most of the atmospheres under study.
\end{abstract}

Key words: methods: data analysis - methods: statistical - techniques: spectroscopic

\section{INTRODUCTION}

The discovery of over 3000 exoplanets has unveiled a large and diverse population. We see planets in a range of sizes, temperatures, and orbits, far exceeding the diversity found in our own solar system. Today, emphasis in the field of exoplanets is shifting from the discovery to the characterization of these exoplanetary bodies, as understanding their nature will in turn provide important clues on the planets' formation and evolution history.

The study of exoplanetary atmospheres represents one of the most immediate and direct ways to characterize exoplanets. To date, the atmospheres of several tens of giant planets, subNeptunes, and super-Earths have been studied and characterized with the Hubble Space Telescope (HST; Charbonneau et al. 2002; Tinetti et al. 2010; Swain et al. 2013; Kreidberg et al. 2014; Sing et al. 2016; Tsiaras et al. 2016), the Spitzer Space Telescope (Grillmair et al. 2007; Knutson et al. 2007, 2011; Tinetti et al. 2007; Charbonneau et al. 2008; Beaulieu et al. 2010; Stevenson et al. 2010; Deming et al. 2011; Todorov et al. 2013), and other ground-based facilities (Redfield et al. 2008; Snellen et al. 2008; Swain et al. 2010; Waldmann et al. 2012; Bean et al. 2013; Brogi et al. 2014; Danielski et al. 2014; Zellem et al. 2014).

With the imminent launch of the James Webb Space Telescope (JWST), it has become fundamental to assess whether the current methods used to interpret these spectra will still be valid when higher-quality data sets will be available. In this work we aim to answer this question in part, exploring the biases induced by common assumptions used in atmospheric retrievals.
One of the major limitations of current observations is the limited wavelength coverage. The best-quality data sets, which led to the confident detection of water vapor in several hot Jupiters and warm Neptunes, have been mainly obtained with the Wide Field Camera 3 on board HST, covering the spectral range 1.1-1.7 $\mu \mathrm{m}$. Nevertheless, it is at longer wavelengths that most rovibrational transitions of molecular species occur. While the Spitzer Space Telescope has given some insight into the longer-wavelength regime to several tens of close-in hot Jupiters, the data have relatively large uncertainties, and they are mostly only photometric measurements. Significant advances in the field of atmospheric characterization can therefore only happen if high-quality observations extending to the longer-wavelength regime are obtained.

In this scenario, the JWST will undoubtedly revolutionize the field of exoplanetary atmospheres, addressing two major problems affecting current observations: wavelength coverage and instrument sensitivity. With a scheduled launch for 2018 October, the large spectral coverage $(0.7-28 \mu \mathrm{m})$ covered by its multiple instruments, combined with high sensitivity and a high degree of instrumental characterization and calibration, will ensure a significant advance in atmospheric characterization (Beichman et al. 2014; Barstow et al. 2015; Batalha et al. 2015; Cowan et al. 2015; Barstow \& Irwin 2016; Greene et al. 2016).

Atmospheric spectra of transiting exoplanets in a broad spectral range will enable us to constrain the abundances of different molecular species, the temperature structure of the atmosphere, and the presence or absence of clouds and hazes. In the case of warm $\mathrm{H} / \mathrm{He}$-dominated atmospheres, one of the key elemental ratios that we aim to constrain is the carbon-to- 
oxygen ratio $(\mathrm{C} / \mathrm{O})$. Such measurements will enable us to distinguish between different formation and migration scenarios, so far poorly constrained (Madhusudhan et al. 2011b; Öberg et al. 2011; Ali-Dib et al. 2014; Thiabaud et al. 2015). While transmission and emission spectra do not provide direct constraints on the elemental abundances, the measurement of the absolute abundances of O-bearing and C-bearing molecules will provide some constraints on the $\mathrm{C} / \mathrm{O}$ ratio. In particular, the excess carbon and oxygen not locked in $\mathrm{CO}$ will form either oxygen-bearing molecules such as $\mathrm{H}_{2} \mathrm{O}$ in atmospheres with $\mathrm{C} / \mathrm{O}<1$ or, in atmospheres with $\mathrm{C} / \mathrm{O}>1$, carbon-rich species such as $\mathrm{HCN}, \mathrm{C}_{2} \mathrm{H}_{2}$, and $\mathrm{CH}_{4}$ (Madhusudhan 2012; Moses et al. 2013a, 2013b; Venot et al. 2015). Determining the atmospheric abundances of these gases in hot Jupiters with high accuracy is therefore paramount, and JWST will give us direct access to absorption features of these molecules in both emission and transmission.

Determining the absolute abundances of atmospheric gases from atmospheric spectra requires the use of retrieval methods. Atmospheric retrieval techniques are now commonly used to infer the properties of exoplanetary atmospheres, including molecular abundances and temperature profiles (Irwin et al. 2008; Madhusudhan \& Seager 2009; Lee et al. 2011; Madhusudhan et al. 2011a; Benneke \& Seager 2012, 2013; Line et al. 2012, 2013, 2014; de Wit \& Seager 2013; Waldmann et al. 2015a, 2015b). These tools enable us to fully map the likelihood space of atmospheric models and to place upper limits and constraints on the abundances of molecules and temperature profiles.

The lack of high signal-to-noise ratio and broad wavelength coverage observations has, however, led current retrievals and forward models to make several assumptions and approximations to reduce the parameter space. The forward model included in most retrieval methods is a 1D radiative transfer model (Brown 2001; Liou 2002; Seager 2011; Tinetti et al. 2012; Hollis et al. 2013), implementing opacity cross sections for the major molecular absorbers, Rayleigh scattering, and collision-induced absorption. Transmission spectra are usually retrieved assuming constant-with-altitude temperature and molecular abundances. This might be a fair approximation when probing narrow wavelength ranges, but can lead to significant biases when larger wavelength ranges are probed. One of the pressing questions we are facing today is whether these assumptions will still be valid in the era of JWST.

In this paper, we aim to address these issues. We study the biases and degeneracies of atmospheric retrievals of highquality, broad wavelength range transmission spectra of hot Jupiters, such as those that will be obtained with instruments on board JWST. We apply and compare different retrieval approaches to synthetic observations for a range of hot atmospheres with different $\mathrm{C} / \mathrm{O}$ computed using photochemical models, and we study the biases of common assumptions used in today's retrievals.

This study aims at answering the following questions:

(a) Are our retrieval approaches and forward models appropriate for the high signal-to-noise ratio and broader wavelength range spectra expected from future facilities such as JWST?

(b) Can we confidently retrieve absolute molecular abundances and infer the $\mathrm{C} / \mathrm{O}$ ratio?

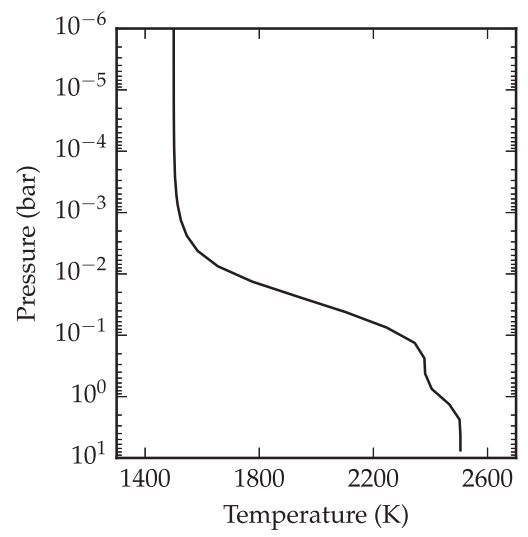

Figure 1. TP profile used for the atmospheres under study.

In Section 2 we describe the chemical and radiative transfer models used to generate the synthetic transmission spectra. We also present the JWST synthetic observations and describe the two retrieval approaches used to interpret these synthetic observations. In Section 3 we describe qualitatively the simulated transmission spectra and present the results of the retrievals. In Section 4 we discuss our results, and in Section 5 we summarize the main conclusions of this study.

\section{METHOD}

\subsection{Chemical Models}

The 1D atmospheric chemical models were generated using the photochemical model developed for hot atmospheres (Venot et al. 2012, and references therein). These models have been used to study exoplanets (Agúndez et al. 2014; Venot et al. 2014, 2015; Venot \& Agúndez 2015; Tsiaras et al. 2016), as well as solar system giant planets (Cavalié et al. 2014; Mousis et al. 2014). The chemical scheme has been developed with combustion specialists and validated in a wide range of pressures (0.001-100 bars) and temperatures (300-2500 K), making this model one of the currently most reliable chemical schemes (Battin-Leclerc et al. 2006; Bounaceur et al. 2007; Anderlohr et al. 2010; Wang et al. 2010). Venot et al. (2015) showed that the use of more complete chemical models, including species with up to six carbon atoms, has little effect on the synthetic spectra. We therefore used the simpler, and computationally faster, scheme that includes species with up to four carbon atoms and is able to model the kinetic behavior of species with up to two carbon atoms. This scheme includes 105 neutral species and 960 reactions (and their reverse reactions). We used a constant diffusion coefficient, $K_{\mathrm{zz}}=10^{8} \mathrm{~cm}^{2} \mathrm{~s}^{-1}$, due to the uncertainties on the vertical mixing acting in exoplanet atmospheres. A similar value has been often used in the literature (Lewis et al. 2010; Line et al. 2011; Moses et al. 2011; Venot et al. 2013). We note, however, that this value might be too high, as pointed out by Parmentier et al. (2013).

We used a temperature-pressure (TP) profile with a highaltitude temperature of $1500 \mathrm{~K}$. The vertical profile is the same as the one used in Venot et al. (2015). It was computed using the analytical model of Parmentier \& Guillot (2014), using coefficients from Parmentier et al. (2015) and the opacities from Valencia et al. (2013). The profile, shown in Figure 1, was obtained by setting the irradiation temperature to $2300 \mathrm{~K}$ and the internal temperature $T_{\text {int }}=100 \mathrm{~K}$. We assumed a planet with $R_{p}=1.162_{J}$ and $M_{p}=1.138 M_{J}$. 
We computed chemical models for an atmosphere of solar metallicity with $\mathrm{C} / \mathrm{O}$ of $0.5,0.7,0.9,1.0,1.1,1.3$, and 1.5 .

\subsection{Synthetic High-resolution Transmission Spectra}

High-resolution $(R \approx 10,000)$ synthetic transmission spectra were computed using the forward models included in TauREx (Waldmann et al. 2015b). This forward model is based on a 1D radiative transfer model that calculates the optical path through the planetary atmosphere. It results in a transmission spectrum of transit depth, i.e., $\left(R_{p} / R_{*}\right)^{2}$, as a function of wavelength. The temperature profile used is the same as the one used for the computation of the photochemical models (Figure 1). We include a precise computation of the pressure-altitude profile and take into account the effect of gravity, temperature, and mean molecular weight in the computation of the scale height in each of the 100 atmospheric layers included in the model. We compute the pressure grid from $10^{-4}$ to 10 bars and define the 10 bar pressure radius to be $R_{p}=1.162 R_{J}$. The mass is set to $M_{p}=1.138 M_{J}$. Among the 105 molecules considered in the photochemical model, we only consider the following seven molecules in the computation of the opacity in the synthetic spectra: $\mathrm{C}_{2} \mathrm{H}_{2}, \mathrm{CH}_{4}, \mathrm{CO}, \mathrm{CO}_{2}, \mathrm{H}_{2} \mathrm{O}, \mathrm{HCN}$, and $\mathrm{NH}_{3}$. We found that among the complete set of 105 molecules contained in the chemical model, these are the most abundant ones in all cases and will therefore dominate the spectral modulation. The wavelength-dependent cross sections for these absorbing molecules were computed using line lists from ExoMol (Barber et al. 2006, 2014; Harris et al. 2006; Yurchenko et al. 2011, 2013; Tennyson \& Yurchenko 2012), HITRAN (Rothman et al. 2013), and HITEMP (Rothman et al. 2010). Note that the mean molecular weight of each atmospheric layer is coupled to the mixing ratio of all 105 molecules. We included additional opacity from Rayleigh scattering of $\mathrm{H}_{2}$ and from collisioninduced absorption of $\mathrm{He}$ and $\mathrm{H}_{2}-\mathrm{H}_{2}$ and $\mathrm{H}_{2}-\mathrm{He}$ pairs (Richard et al. 2012).

\subsection{JWST Spectra}

We simulated spectra for the Near-Infrared Imager and Slitless Spectrograph (NIRISS) in Single-Object Slitless Spectroscopy (SOSS) mode using the GR700XD optics (Doyon et al. 2012). We applied a lower-wavelength cutoff at $1 \mu \mathrm{m}$ to avoid saturation and a long-wavelength cutoff at $2.5 \mu \mathrm{m}$ to avoid spectral contamination (Greene et al. 2016). We then used the Near Infrared Camera (NIRCam) using the long-wavelength (LW) channel and the F322W2 and F444W filters, covering the $2.5-3.9 \mu \mathrm{m}$ and $3.9-5.0 \mu \mathrm{m}$ spectral ranges, respectively (Greene et al. 2007). An alternative could be the use of the Near-Infrared Spectrograph (NIRSpec) in its high-resolution mode with the two instrumental configurations: F170LP/G235H $(1.7-3.1 \mu \mathrm{m})$ and F290LP/G395H $(2.9-5.2 \mu \mathrm{m})$ (Ferruit et al. 2014). Finally, we use the MidInfrared Instrument (MIRI) to cover the 5.0-10.0 $\mu \mathrm{m}$ wavelength range. We use MIRI in slitless mode, using the Low Resolution Spectrometer (LRS), and we apply a longwavelength cutoff of $10 \mu \mathrm{m}$ due to the degrading signal-tonoise ratio at longer wavelengths (Kendrew et al. 2015). Each observation covering the full wavelength range of $1-10 \mu \mathrm{m}$ will therefore require four separate observations. We have considered a $1 \mathrm{hr}$ effective integration time during the transit and the same amount of time on the star alone. For each mode, the same amount of time was used. Table 1 summarizes the instrument modes considered in this study.

The noise in the spectra was calculated taking into account the star photon noise, the zodiacal and telescope background noise (integrated over the entire band pass of the spectrometer for the slitless mode), and the detector dark current and noise. We assumed a star similar to HD 189733. The star spectrum used was generated using the PHOENIX atmosphere star code (Husser et al. 2013). For NIRISS and NIRCAM, we have binned the spectra to a constant spectral resolution of $R=100$. For such a bright star we realized that we are in fact very close to the limitation from systematics of the JWST. Such systematics are difficult to assess, but we can reasonably assume that they will be lower than HST. Given the latest performances achieved with $H S T$ (e.g., Tsiaras et al. 2016), we can anticipate that the systematics for NIRISS and NIRCAM will be better than about $20 \mathrm{ppm}$. For MIRI, Greene et al. (2016) adopted a value of $50 \mathrm{ppm}$, and Beichman et al. (2014) took a value of $30 \mathrm{ppm}$; we have adopted an intermediate value of $40 \mathrm{ppm}$. An example of a final spectrum is shown in Figure 2.

\subsection{Atmospheric Retrieval}

The analysis and interpretation of the simulated observed spectra were carried out using TauREx (Waldmann et al. 2015b). Recently, TauREx has been used to model the spectra obtained with the Wide Field Camera 3 on board HST for HD 209458b and $55 \mathrm{Cnc}$ e (Tsiaras et al. 2015, 2016).

Two retrieval approaches were used as part of the current study. Both approaches did not assume any prior knowledge on the chemistry, i.e., the absolute abundance of all gases taken into account is fitted independently. The only difference between the two approaches is in the parameterization of the temperature profile:

1. In the first case we assumed an isothermal TP profile. We will refer to this method as "TP-ISO." This approach is the most commonly used when fitting transmission spectra (Irwin et al. 2008; Benneke \& Seager 2012; Line et al. 2012) and includes a parameterization of the atmosphere assuming constant-with-altitude mixing ratio and temperature profiles. Crucially, it does not assume any prior on the chemistry of the atmosphere. The free parameters of the retrieval were the absolute abundance of each atmospheric constituent taken into account, the isothermal temperature, the cloud parameters, and the $10 \mathrm{bar}$ pressure radius. The mean molecular weight is coupled to the fitted composition, and we assumed the bulk atmosphere to be formed by a mixture of hydrogen and helium, whose ratio is fixed to the solar value $\left(85 \% \mathrm{H}_{2}\right.$ and $15 \% \mathrm{He})$. We assumed uniform priors in log space for the absolute abundances, ranging from $10^{-12}$ to 1 . We assumed uniform priors for the temperature (1300-2500 $\mathrm{K})$ and for the 10 bar radius $\left(1.05-1.28 R_{\mathrm{Jup}}\right)$. The prior width of the $10 \mathrm{bar}$ radius was determined by assuming a relative uncertainty on $R_{p}$ of $20 \%\left(R_{p}=1.162 R_{J}\right)$. Lastly, we fitted the cloud top pressure with a uniform prior in log space $\left(10^{-5}-10\right.$ bars $)$. This parameterization resulted in 10 free variables.

2. In the second case, we assumed a more complex TP profile described by five separate parameters. We will refer to this method as "TP-PARAM." Since the 


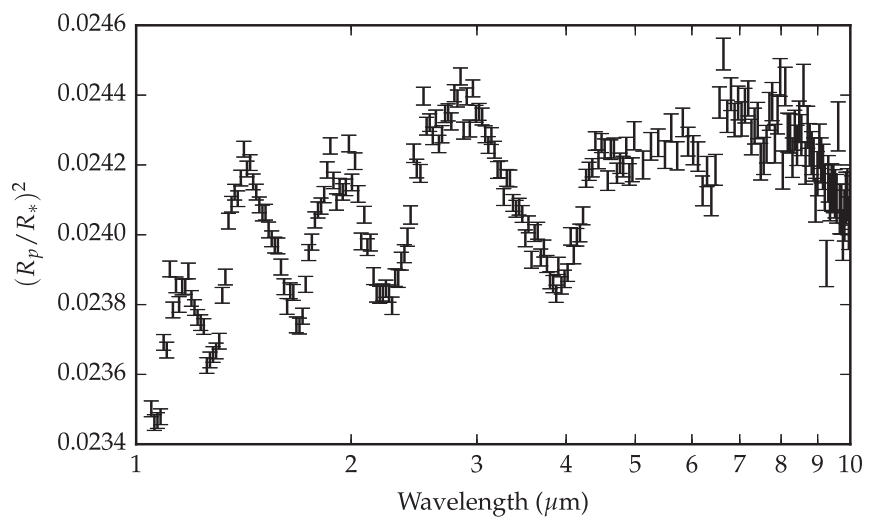

Figure 2. Simulated JWST observation for $\mathrm{C} / \mathrm{O}=0.5$. The spectrum was obtained combining four separate synthetic observations obtained with NIRISS, NIRCam, and MIRI to cover the $1-10 \mu \mathrm{m}$ spectral range. This spectrum would therefore require observing a total of four transits.

temperature profile of the atmospheres under study is highly nonisothermal for pressures greater than 1 mbar (see Figure 1), fitting an isothermal profile might lead to biases. We therefore investigated the effectiveness of fitting a more complex profile using this second method. We used the parameterization of Guillot (2010) modified by Line et al. (2013) and Parmentier \& Guillot (2014). There are five parameters that define the temperature profile: the planet internal heat flux $\left(T_{\text {int }}\right)$, the stellar irradiation flux $\left(T_{\text {irr }}\right)$, the opacities in the optical and infrared $\left(\kappa_{\nu 1}, \kappa_{\nu 2}\right)$, and a weighting factor between optical opacities $(\alpha)$. For a full description of this model we refer the reader to Section 3.1 of Line et al. (2013). These five parameters replace the single parameter used for the isothermal profile in the first method. This model only differs from the first one for the type of TP profile used. This parameterization resulted in 14 free variables.

The parameterized profile described above is commonly used in the retrieval of emission spectra, where the spectral features are more sensitive to temperature gradients than in transmission. It has received little attention in the retrieval of transmission spectra, as it is assumed that transmission spectra are much less sensitive to temperature gradients, and therefore isothermal profiles, thought to represent the "average" atmospheric temperature, have always been used. Previous studies have addressed the potential bias of the isothermal assumption (Barstow et al. 2013) and found that some information on the temperature profile could be retrieved in transmission only in the highest signal-to-noise ratio and broad wavelength coverage cases.

We used these two approaches to interpret the synthetic JWST observations in a range of $\mathrm{C} / \mathrm{O}$. In all cases we used the MultiNest sampling algorithm (Feroz \& Hobson 2008) to finely sample the parameter space and obtain the posterior distributions of the model parameters. We chose this method instead of a more classical MCMC, as MultiNest can better map the likelihood of highly degenerate parameter spaces. Table 2 summarizes the free parameters and the corresponding prior widths used in the two retrieval methods.
Table 1

JWST Instrument Modes

\begin{tabular}{llc}
\hline \hline Instrument & Mode & Wavelength Range $(\mu \mathrm{m})$ \\
\hline NIRISS & SOSS/GR700XD & $1.0-2.5 \mu \mathrm{m}$ \\
NIRCam & LW grism/F322W2 & $2.5-3.9 \mu \mathrm{m}$ \\
NIRCam & LW grism/F444W & $3.9-5.0 \mu \mathrm{m}$ \\
MIRI & Slitless/LRS prism & $5.0-10.0 \mu \mathrm{m}$ \\
\hline
\end{tabular}

\section{RESULTS}

\subsection{Chemical Models and Transmission Spectra}

Figure 3 shows the vertical abundance profiles of seven molecules for all $\mathrm{C} / \mathrm{O}$ ratios considered in this study, and Figure 4 shows the synthetic transmission spectra and contributions of the major opacity sources for the same $\mathrm{C} / \mathrm{O}$ values. It can be clearly seen that the chemistry and the resulting spectra change significantly between $\mathrm{C} / \mathrm{O}<1$, $\mathrm{C} / \mathrm{O}=1$, and $\mathrm{C} / \mathrm{O}>1$.

First, we note that while the transmission spectra of an oxygen-rich atmosphere are dominated almost entirely by $\mathrm{H}_{2} \mathrm{O}$, with additional features from $\mathrm{CO}$ at $4.6 \mu \mathrm{m}$ and from $\mathrm{CO}_{2}$ at $4.3 \mu \mathrm{m}$, a carbon-rich atmosphere is dominated by $\mathrm{HCN}$ and $\mathrm{CH}_{4}$, with additional features from $\mathrm{CO}$ at $4.6 \mu \mathrm{m}$ and $\mathrm{C}_{2} \mathrm{H}_{2}$ at $1.7,3.0$, and $7.5 \mu \mathrm{m}$. At the $\mathrm{C} / \mathrm{O}=1.0$ threshold the transmission spectrum is dominated by $\mathrm{H}_{2} \mathrm{O}$ and $\mathrm{HCN}$ and exhibits strong features of $\mathrm{CO}$ at 2.3 and $4.6 \mu \mathrm{m}$. Weak features from $\mathrm{CH}_{4}$ are also seen at 3.4 and $7.6 \mu \mathrm{m}$. Tight constraints on the abundances of all these molecules are therefore paramount to constrain the chemistry and $\mathrm{C} / \mathrm{O}$ of these atmospheres.

Between $\mathrm{C} / \mathrm{O}=0.5$ and 0.9 we see a gradual decrease in the molar fractions of $\mathrm{H}_{2} \mathrm{O}$ and $\mathrm{CO}_{2}$ and a slight increase in the $\mathrm{CH}_{4}, \mathrm{HCN}$, and $\mathrm{C}_{2} \mathrm{H}_{2}$ abundances, while $\mathrm{CO}$ remains relatively constant. The resulting transmission spectra in this $\mathrm{C} / \mathrm{O}$ range show the progressive decrease in the absorption of $\mathrm{H}_{2} \mathrm{O}$ (which remains the dominant absorber across this $\mathrm{C} / \mathrm{O}$ range) and the resulting emergence of $\mathrm{CO}$, while all the other molecules remain hidden. It is only at $\mathrm{C} / \mathrm{O}>1.0$ that $\mathrm{HCN}$ is sufficiently abundant to be clearly seen in the transmission spectrum. We note that at this threshold we see the minimum average absorption from active gases across most of the spectrum, so that in some regions we can also see the emergence of the collision-induced absorption from $\mathrm{H}_{2}-\mathrm{H}_{2}$ and $\mathrm{H}_{2}-\mathrm{He}$ pairs.

At $\mathrm{C} / \mathrm{O}=1.1$ the $\mathrm{H}_{2} \mathrm{O}$ and $\mathrm{CO}_{2}$ content drastically drops, while the abundances of $\mathrm{CH}_{4}, \mathrm{HCN}$, and $\mathrm{C}_{2} \mathrm{H}_{2}$ increase significantly. The corresponding transmission spectra show features of $\mathrm{CH}_{4}, \mathrm{HCN}, \mathrm{CO}$, and $\mathrm{C}_{2} \mathrm{H}_{2}$. At progressively higher $\mathrm{C} / \mathrm{O}$ ratios we see the increase in abundance of $\mathrm{CH}_{4}, \mathrm{HCN}$, and $\mathrm{C}_{2} \mathrm{H}_{2}$ and the progressive decrease of $\mathrm{CO}$ abundance. However, we note that the resulting spectra are very similar to each other. The only differences in the spectra are the weakening of $\mathrm{CO}$ at $4.6 \mu \mathrm{m}$ and the strengthening of $\mathrm{C}_{2} \mathrm{H}_{2}$ at 3 and $7.5 \mu \mathrm{m}$.

Finally, we note that $\mathrm{C}_{2} \mathrm{H}_{2}$ might actually have additional and much stronger features than those seen here. This is because the line list used for this molecule comes from HITRAN and has been computed experimentally at Earth-like temperatures. It is therefore suboptimal to use this line list for such high temperatures $(>1500 \mathrm{~K})$. As an appropriate hot line list would include many more transitions resulting from the population of higher vibrational levels, additional spectral features (i.e., "hot bands") are expected, together with the strengthening of the features that can already be seen at lower 
Table 2

Free Parameters of the Two Retrieval Approaches Used in This Study

\begin{tabular}{llcl}
\hline \hline Approach & Parameter & Prior & Description \\
\hline TP-ISO & $\log \mathrm{H}_{2} \mathrm{O}, \log \mathrm{CO}, \log \mathrm{CO}_{2}$, & Molecular abundances \\
(10 free parameters) & $\log \mathrm{CH}_{4} \log \mathrm{NH}_{3}, \log \mathrm{HCN}$, & $-12 \ldots 1$ & Isothermal temperature \\
& $\log \mathrm{C}_{2} \mathrm{H}_{2}$ & & Planetary radius at 10 bars \\
& $T_{\text {iso }}(\mathrm{K})$ & $1300 \ldots 2600$ & Cloud top pressure \\
& $R_{p}\left(R_{\mathrm{Jup}}\right)$ & $1.05 \ldots 1.28$ & Molecular abundances \\
& $\log \left(P_{\mathrm{top}}(\mathrm{Pa})\right)$ & $0 \ldots 6$ & \\
\hline TP-PARAM & $\log \mathrm{H}_{2} \mathrm{O}, \log \mathrm{CO}, \log \mathrm{CO}_{2}$, & Stellar flux at the top of the atmosphere \\
$(14$ free parameters $)$ & $\log \mathrm{CH}_{4} \log \mathrm{NH}_{3}, \log \mathrm{HCN}$, & Mean infrared opacity \\
& $\log \mathrm{C}_{2} \mathrm{H}_{2}$ & $12 \ldots 1$ & Optical opacity sources \\
& $T_{\text {irr }}(\mathrm{K})$ & $-4 \ldots 1$ & Weighting factor for $\kappa_{\nu 1}$ and $\kappa_{\nu 2}$ \\
& $\log \kappa_{\mathrm{IR}}$ & $-4 \ldots 1$ & Planetary radius at 10 bars \\
& $\log \kappa_{\nu 1}, \log \kappa_{\nu 2}$ & $0 \ldots 1$ & Cloud top pressure \\
\hline
\end{tabular}

Note. The TP-ISO approach refers to the retrieval using an isothermal TP profile, while the TP-PARAM refers to the retrieval using a parameterized TP profile.
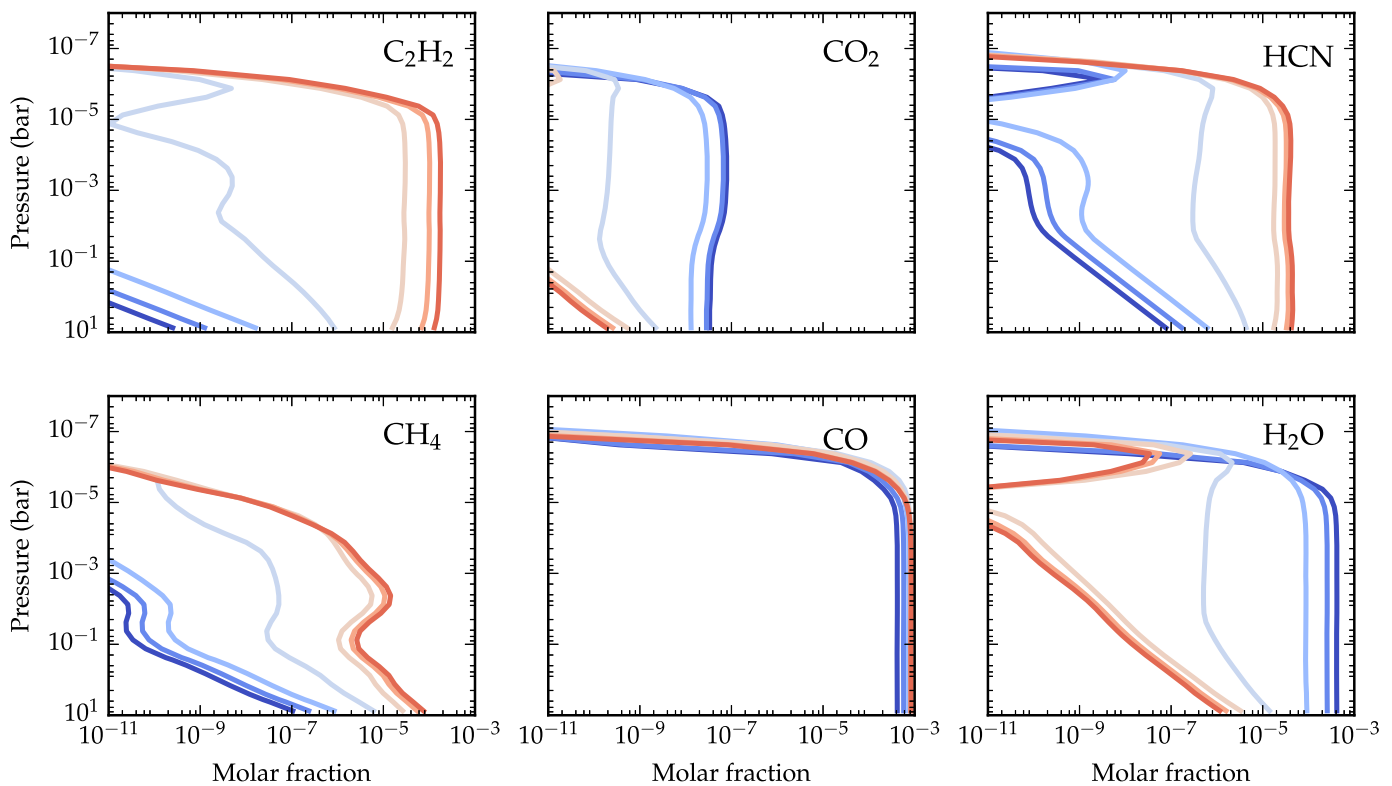

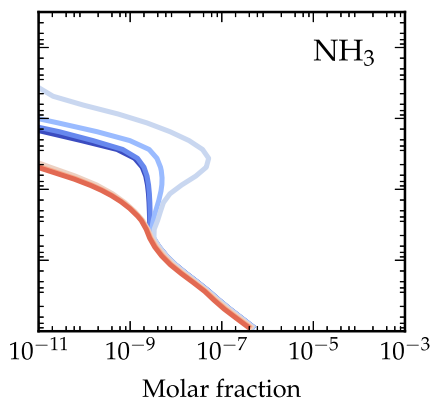

Figure 3. Vertical abundance profiles for different molecules for a range of $\mathrm{C} / \mathrm{O}$. The different colored lines show the molar fraction profiles at different $\mathrm{C} / \mathrm{O}$, as shown by the legend.

temperatures. Such a list is under development at $\mathrm{ExoMol}^{5}$ (private communication).

\subsection{Retrieval of Temperature Profiles}

Figure 5 shows the retrieved temperature profiles using the two approaches for all $\mathrm{C} / \mathrm{O}$ values. It can be clearly seen that in most cases the retrieved TP profile is within $1 \sigma$ of the input profile using the TP-PARAM method, while using the TP-ISO method the input profile is almost entirely outside the $1 \sigma$ retrieved error bars.

For $\mathrm{C} / \mathrm{O}>1$ (first three panels), it can be seen how the TPPARAM method fits both the upper atmosphere temperature and the lower-altitude part of the atmosphere. We found that the upper atmospheric temperature could be well fitted within about $1 \sigma-3 \sigma$ using the parameterized TP profile in all cases.

\footnotetext{
5 http://www.exomol.com
}

The high-altitude temperature was found to be $T=1502 \pm 66 \mathrm{~K}, T=1425 \pm 27 \mathrm{~K}$, and $T=1433 \pm 50 \mathrm{~K}$ for $\mathrm{C} / \mathrm{O}=0.5,0.7$, and 0.9 , respectively. Using the TP-ISO method, the retrieved temperatures for the same $\mathrm{C} / \mathrm{O}$ values were $\quad T=1572 \pm 14 \mathrm{~K}, \quad T=1610 \pm 17 \mathrm{~K}, \quad$ and $T=1716 \pm 24 \mathrm{~K}$, respectively. In all cases, the input profile has a high-altitude temperature of $1500 \mathrm{~K}$.

From these plots we can also appreciate that the nonisothermal part of the profile could be fitted within $1 \sigma$ for $\mathrm{C} / \mathrm{O}=0.5,0.7$, and 0.9 . Interestingly, we also note that for $\mathrm{C} / \mathrm{O}<1$ the constraint of the low-altitude temperature $\left(P>10^{-3}\right.$ bars $)$ improves for higher $\mathrm{C} / \mathrm{O}$, while the fit of the high-altitude part of the profile $\left(P>10^{-3}\right.$ bars $)$ improves for lower $\mathrm{C} / \mathrm{O}$.

The last three panels in Figure 5 show the retrieved temperature profiles using both approaches for $\mathrm{C} / \mathrm{O}>1$. We can see that the TP-ISO approach retrieves a temperature of 

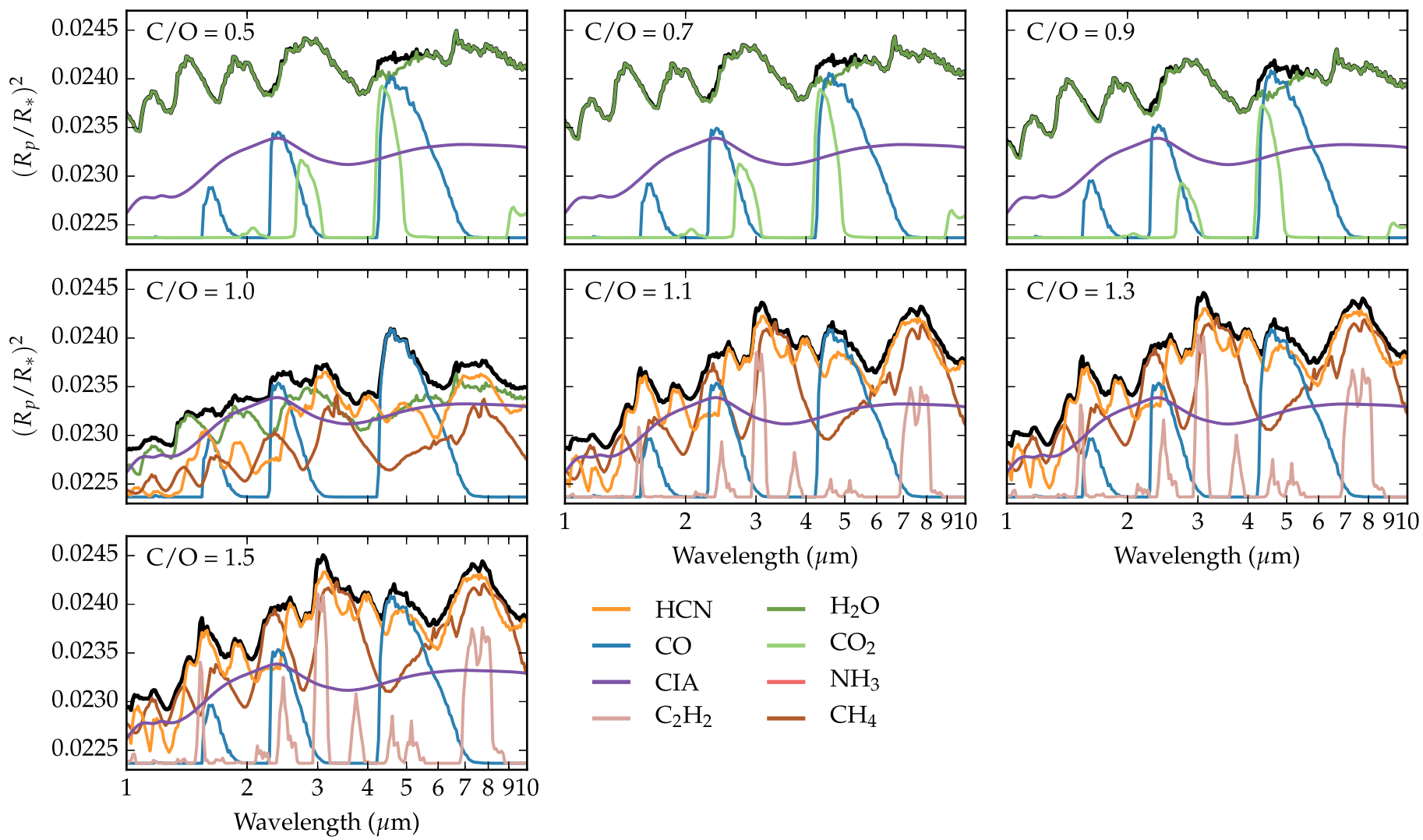

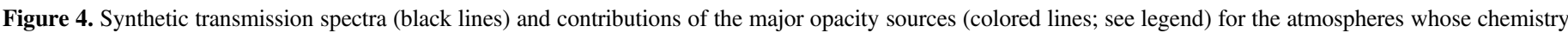

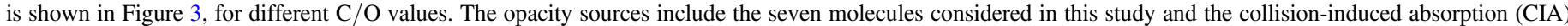

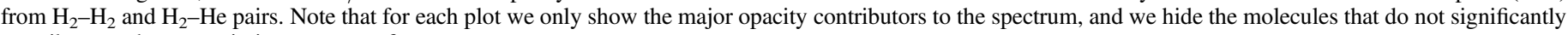
contribute to the transmission spectrum features.
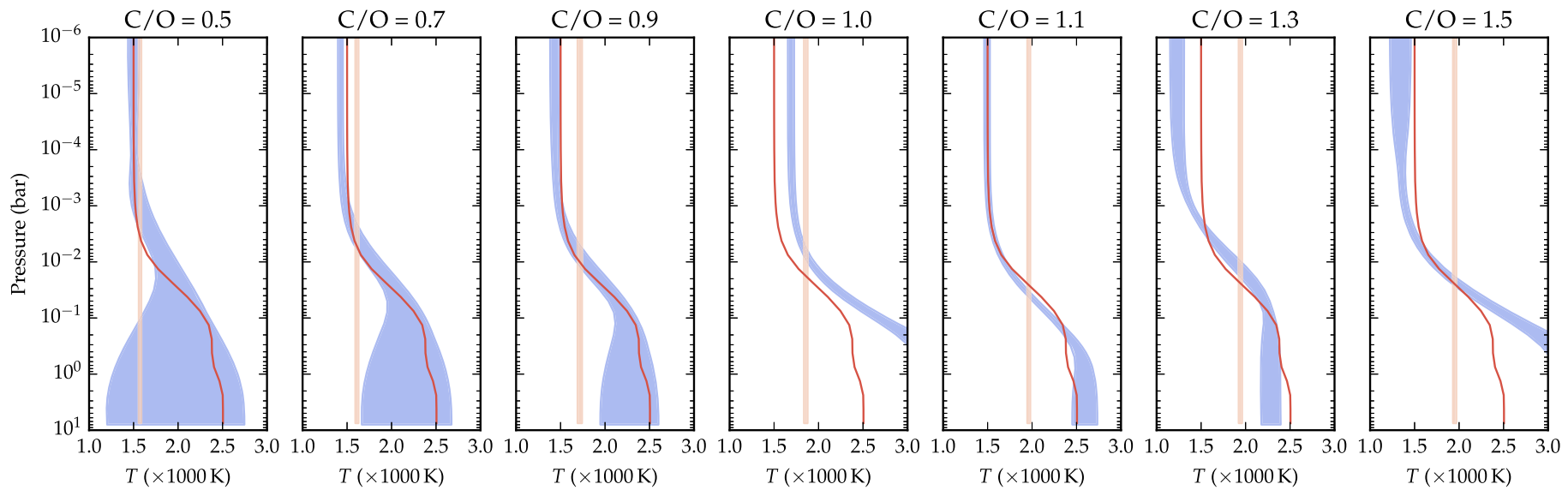

Figure 5. Retrieved temperature profiles for the approach with isothermal profile (pink) and parameterized profile (blue) for different $\mathrm{C} / \mathrm{O}$. The red line shows the input profile. The shaded areas show the $1 \sigma$ confidence level.

$\approx 2000 \mathrm{~K}$, with an uncertainty of $\approx 20 \mathrm{~K}$ in all cases. Using the parameterized approach, we could fit the high-altitude temperature within about $1 \sigma$ for $\mathrm{C} / \mathrm{O}=1.1$ and 1.5 and within $3.4 \sigma$ for $\mathrm{C} / \mathrm{O}=1.3$. We also note that while the low-altitude part of the TP profile for $\mathrm{C} / \mathrm{O}=1.1$ and 1.3 is well constrained within about $1 \sigma$, for $\mathrm{C} / \mathrm{O}=1.5$ the fit is poor for pressures higher than 0.1 bars.

For $\mathrm{C} / \mathrm{O}=1$ we note that the TP profile is poorly retrieved, with the TP-PARAM method giving slightly better results. In both cases, however, the input profile cannot be retrieved within several sigma: the retrieved upper atmosphere temperature is $6 \sigma$ and $18 \sigma$ away from the true state using the TP-PARAM and TP-ISO methods, respectively. Additionally, the lower atmosphere temperature $(P<0.1$ bars $)$ is not retrieved in both cases.

\subsection{Retrieval of Atmospheric Abundances}

The atmospheric retrieval results for the atmospheric abundances of $\mathrm{H}_{2} \mathrm{O}, \mathrm{CO}, \mathrm{CO}_{2}, \mathrm{CH}_{4}, \mathrm{HCN}, \mathrm{C}_{2} \mathrm{H}_{2}$, and $\mathrm{NH}_{3}$ are shown in Table 3 and in Figures 6 and 7. In these plots, the input mixing ratios for each molecule at each $\mathrm{C} / \mathrm{O}$ are also shown with solid lines as a function of pressure. We note again 
Table 3

Retrieved Absolute Abundances with $1 \sigma$ Uncertainty for the Seven Molecules and Seven C/O Values Considered in This Study

\begin{tabular}{|c|c|c|c|c|}
\hline \multirow{2}{*}{ Parameter } & \multirow[t]{2}{*}{$\mathrm{C} / \mathrm{O}$} & \multirow{2}{*}{$\begin{array}{l}\text { Input Value } \\
\text { (at } 0.1 \text { bars) }\end{array}$} & \multicolumn{2}{|c|}{ Retrieved Value } \\
\hline & & & TP-ISO & TP-PARAM \\
\hline \multirow[t]{7}{*}{$\overline{\mathrm{H}_{2} \mathrm{O}}$} & 0.5 & $4.08 \times 10^{-4}$ & $4.66 \times 10^{-3}-6.53 \times 10^{-3}(15.4)$ & $3.73 \times 10^{-4}-1.98 \times 10^{-2}(1.0)$ \\
\hline & 0.7 & $2.52 \times 10^{-4}$ & $2.98 \times 10^{-3}-4.12 \times 10^{-3}(16.2)$ & $1.82 \times 10^{-4}-5.37 \times 10^{-4}(0.4)$ \\
\hline & 0.9 & $8.63 \times 10^{-5}$ & $6.61 \times 10^{-4}-8.18 \times 10^{-4}(20.1)$ & $4.84 \times 10^{-5}-9.75 \times 10^{-5}(0.3)$ \\
\hline & 1.0 & $1.20 \times 10^{-6}$ & $1.63 \times 10^{-6}-1.94 \times 10^{-6}(4.6)$ & $1.43 \times 10^{-6}-1.62 \times 10^{-6}(4.0)$ \\
\hline & 1.1 & $4.56 \times 10^{-8}$ & $<1.04 \times 10^{-8}(1.4)$ & $<5.44 \times 10^{-12}(6.2)$ \\
\hline & 1.3 & $2.44 \times 10^{-8}$ & $<5.13 \times 10^{-9}(1.5)$ & $<2.58 \times 10^{-8}(1.1)$ \\
\hline & 1.5 & $1.85 \times 10^{-8}$ & $<5.74 \times 10^{-9}(1.3)$ & $<1.49 \times 10^{-8}(0.8)$ \\
\hline \multirow[t]{7}{*}{$\mathrm{CO}$} & 0.5 & $4.13 \times 10^{-4}$ & $8.74 \times 10^{-3}-1.10 \times 10^{-2}(28.0)$ & $3.64 \times 10^{-4}-5.33 \times 10^{-2}(0.9)$ \\
\hline & 0.7 & $5.70 \times 10^{-4}$ & $9.46 \times 10^{-3}-1.17 \times 10^{-2}(27.0)$ & $2.69 \times 10^{-4}-1.17 \times 10^{-3}(0.0)$ \\
\hline & 0.9 & $7.34 \times 10^{-4}$ & $1.15 \times 10^{-2}-1.44 \times 10^{-2}(25.4)$ & $2.90 \times 10^{-4}-7.79 \times 10^{-4}(0.4)$ \\
\hline & 1.0 & $8.22 \times 10^{-4}$ & $2.24 \times 10^{-3}-3.38 \times 10^{-3}(5.9)$ & $2.44 \times 10^{-3}-3.40 \times 10^{-3}(7.5)$ \\
\hline & 1.1 & $8.24 \times 10^{-4}$ & $7.39 \times 10^{-3}-9.62 \times 10^{-3}(17.6)$ & $1.01 \times 10^{-3}-1.01 \times 10^{-3}(799.1)$ \\
\hline & 1.3 & $8.24 \times 10^{-4}$ & $8.35 \times 10^{-3}-1.06 \times 10^{-2}(20.7)$ & $6.33 \times 10^{-4}-1.67 \times 10^{-3}(0.5)$ \\
\hline & 1.5 & $8.24 \times 10^{-4}$ & $8.17 \times 10^{-3}-1.03 \times 10^{-2}(20.9)$ & $8.91 \times 10^{-4}-1.26 \times 10^{-3}(1.4)$ \\
\hline \multirow[t]{7}{*}{$\mathrm{CO}_{2}$} & 0.5 & $3.60 \times 10^{-8}$ & $9.46 \times 10^{-7}-1.82 \times 10^{-6}(11.0)$ & $8.63 \times 10^{-8}-4.13 \times 10^{-6}(1.5)$ \\
\hline & 0.7 & $3.07 \times 10^{-8}$ & $5.95 \times 10^{-7}-1.14 \times 10^{-6}(10.2)$ & $4.13 \times 10^{-8}-1.27 \times 10^{-7}(1.5)$ \\
\hline & 0.9 & $1.35 \times 10^{-8}$ & $1.57 \times 10^{-7}-2.77 \times 10^{-7}(9.6)$ & $1.41 \times 10^{-8}-2.87 \times 10^{-8}(1.1)$ \\
\hline & 1.0 & $2.10 \times 10^{-10}$ & $<2.78 \times 10^{-9}$ & $<3.24 \times 10^{-9}(0.3)$ \\
\hline & 1.1 & $8.01 \times 10^{-12}$ & $<3.32 \times 10^{-10}(0.6)$ & $<7.64 \times 10^{-10}(2.5)$ \\
\hline & 1.3 & $4.28 \times 10^{-12}$ & $<7.23 \times 10^{-10}(0.9)$ & $<8.56 \times 10^{-12}(0.4)$ \\
\hline & 1.5 & $3.25 \times 10^{-12}$ & $<4.05 \times 10^{-10}(1.0)$ & $<4.51 \times 10^{-7}(1.3)$ \\
\hline \multirow[t]{7}{*}{$\mathrm{CH}_{4}$} & 0.5 & $6.43 \times 10^{-11}$ & $<1.85 \times 10^{-7}(0.6)$ & $<1.30 \times 10^{-7}(0.7)$ \\
\hline & 0.7 & $1.44 \times 10^{-10}$ & $<2.86 \times 10^{-7}(0.5)$ & $<1.97 \times 10^{-8}(0.3)$ \\
\hline & 0.9 & $5.39 \times 10^{-10}$ & $<2.03 \times 10^{-8}(0.1)$ & $<7.50 \times 10^{-10}(0.5)$ \\
\hline & 1.0 & $4.36 \times 10^{-8}$ & $8.48 \times 10^{-8}-1.13 \times 10^{-7}(5.6)$ & $4.61 \times 10^{-8}-6.35 \times 10^{-8}(1.4)$ \\
\hline & 1.1 & $1.16 \times 10^{-6}$ & $9.23 \times 10^{-6}-1.15 \times 10^{-5}(20.0)$ & $3.06 \times 10^{-6}-3.06 \times 10^{-6}(43189.6)$ \\
\hline & 1.3 & $2.20 \times 10^{-6}$ & $2.03 \times 10^{-5}-2.55 \times 10^{-5}(20.4)$ & $4.85 \times 10^{-6}-8.79 \times 10^{-6}(3.6)$ \\
\hline & 1.5 & $2.92 \times 10^{-6}$ & $1.80 \times 10^{-5}-2.22 \times 10^{-5}(18.6)$ & $5.66 \times 10^{-6}-6.57 \times 10^{-6}(10.0)$ \\
\hline \multirow[t]{7}{*}{$\mathrm{HCN}$} & 0.5 & $1.32 \times 10^{-9}$ & $<2.15 \times 10^{-5}(0.4)$ & $<1.15 \times 10^{-7}(0.0)$ \\
\hline & 0.7 & $2.94 \times 10^{-9}$ & $<2.94 \times 10^{-7}(0.1)$ & $<2.68 \times 10^{-8}(0.4)$ \\
\hline & 0.9 & $1.10 \times 10^{-8}$ & $<5.54 \times 10^{-7}(0.3)$ & $<3.03 \times 10^{-8}(0.9)$ \\
\hline & 1.0 & $8.84 \times 10^{-7}$ & $6.60 \times 10^{-7}-8.20 \times 10^{-7}(1.7)$ & $5.18 \times 10^{-7}-6.14 \times 10^{-7}(5.3)$ \\
\hline & 1.1 & $2.09 \times 10^{-5}$ & $5.53 \times 10^{-5}-6.74 \times 10^{-5}(10.8)$ & $2.18 \times 10^{-5}-2.18 \times 10^{-5}(1355.8)$ \\
\hline & 1.3 & $3.58 \times 10^{-5}$ & $1.20 \times 10^{-4}-1.49 \times 10^{-4}(12.1)$ & $3.00 \times 10^{-5}-5.79 \times 10^{-5}(0.5)$ \\
\hline & 1.5 & $4.42 \times 10^{-5}$ & $1.01 \times 10^{-4}-1.23 \times 10^{-4}(9.2)$ & $3.47 \times 10^{-5}-4.26 \times 10^{-5}(0.3)$ \\
\hline \multirow[t]{7}{*}{$\mathrm{C}_{2} \mathrm{H}_{2}$} & 0.5 & $8.54 \times 10^{-14}$ & $<4.18 \times 10^{-7}$ & $<9.43 \times 10^{-8}(2.2)$ \\
\hline & 0.7 & $4.26 \times 10^{-13}$ & $<5.26 \times 10^{-7}(1.6)$ & $<1.03 \times 10^{-7}(1.8)$ \\
\hline & 0.9 & $5.96 \times 10^{-12}$ & $<2.97 \times 10^{-7}(1.1)$ & $<5.24 \times 10^{-7}(1.3)$ \\
\hline & 1.0 & $3.88 \times 10^{-8}$ & $<4.08 \times 10^{-9}(1.6)$ & $<4.77 \times 10^{-9}(1.6)$ \\
\hline & 1.1 & $2.72 \times 10^{-5}$ & $1.66 \times 10^{-4}-2.88 \times 10^{-4}(7.5)$ & $2.51 \times 10^{-5}-2.51 \times 10^{-5}(0.1)$ \\
\hline & 1.3 & $9.66 \times 10^{-5}$ & $7.19 \times 10^{-4}-1.17 \times 10^{-3}(9.2)$ & $5.76 \times 10^{-5}-4.44 \times 10^{-4}(0.5)$ \\
\hline & 1.5 & $1.68 \times 10^{-4}$ & $9.42 \times 10^{-4}-1.47 \times 10^{-3}(8.7)$ & $1.64 \times 10^{-4}-2.86 \times 10^{-4}(0.9)$ \\
\hline \multirow[t]{7}{*}{$\mathrm{NH}_{3}$} & 0.5 & $9.73 \times 10^{-9}$ & $<1.04 \times 10^{-7}(0.5)$ & $9.74 \times 10^{-12}-3.33 \times 10^{-8}(0.7)$ \\
\hline & 0.7 & $9.73 \times 10^{-9}$ & $<4.43 \times 10^{-7}(0.3)$ & $<1.11 \times 10^{-8}(0.9)$ \\
\hline & 0.9 & $9.65 \times 10^{-9}$ & $<3.53 \times 10^{-6}(10.9)$ & $<9.63 \times 10^{-8}(0.5)$ \\
\hline & 1.0 & $9.62 \times 10^{-9}$ & $<3.76 \times 10^{-7}(1.4)$ & $<9.12 \times 10^{-8}(3.2)$ \\
\hline & 1.1 & $8.70 \times 10^{-9}$ & $<1.80 \times 10^{-6}(36.4)$ & $<2.89 \times 10^{-7}(4651.3)$ \\
\hline & 1.3 & $7.97 \times 10^{-9}$ & $<2.02 \times 10^{-6}(29.5)$ & $<5.93 \times 10^{-7}(5.6)$ \\
\hline & 1.5 & $7.51 \times 10^{-9}$ & $<2.15 \times 10^{-6}(35.7)$ & $<1.76 \times 10^{-7}(1452.3)$ \\
\hline
\end{tabular}

Note. For each retrieved parameter, we show in parentheses how many sigma away the retrieved value is from the true state.

that the retrieved abundances are constant with altitude, so that a single parameter is retrieved for each molecule using both the TP-ISO (left panels) and TP-PARAM (right panels) retrieval methods. Moreover, we found that $\mathrm{NH}_{3}$ is never well retrieved; hence, we do not show its retrieved values in these figures. This is not surprising given that $\mathrm{NH}_{3}$ is never seen in the simulated transmission spectra (Figure 4).

In general, we found that the TP-ISO method retrieves higher abundances by about one order of magnitude and significantly underestimates the error bars, causing strong biases, while the
TP-PARAM method retrieves the correct true state within $1 \sigma$ for all atmospheres with $\mathrm{C} / \mathrm{O}$ greater and less than 1 , but not for $\mathrm{C} / \mathrm{O}=1$. This is also because the retrieved error bars are significantly larger.

Looking at the transmission spectra for $\mathrm{C} / \mathrm{O}<1$ in Figure 4, it can be seen that $\mathrm{H}_{2} \mathrm{O}$ has multiple features across the entire wavelength range and is therefore the dominant molecule. Indeed, we found that, for these $\mathrm{C} / \mathrm{O}$ values, the retrieved abundance of $\mathrm{H}_{2} \mathrm{O}$ has the smallest uncertainties, but only the approach using the parameterized TP profile gives unbiased 

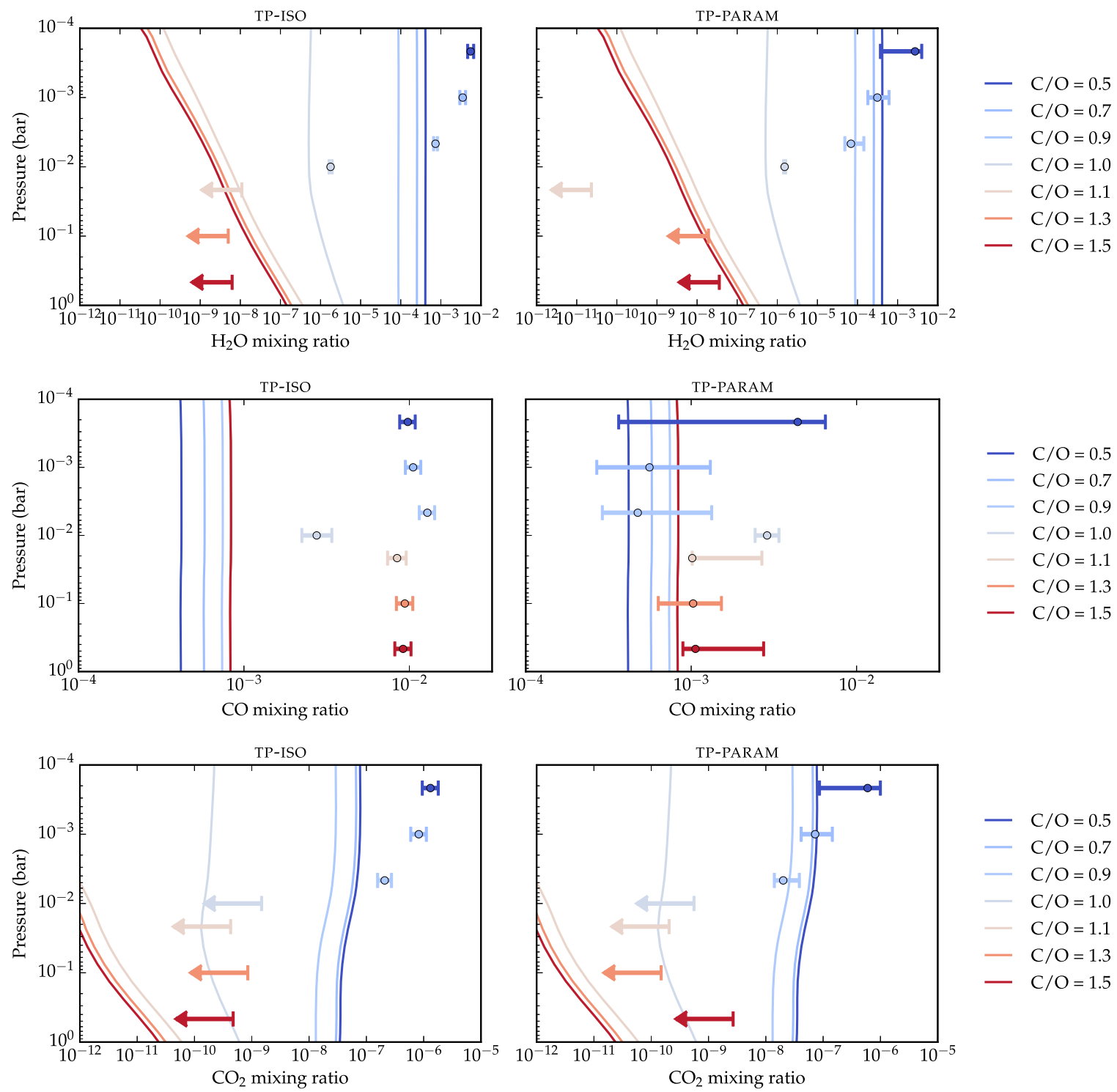

Figure 6. Retrieved $\mathrm{H}_{2} \mathrm{O}$ (top), $\mathrm{CO}$ (middle), and $\mathrm{CO}_{2}$ (bottom) abundance for $\mathrm{C} / \mathrm{O}=0.5-1.5$ using the approach with an isothermal profile (left) and that with a parameterized TP profile (right). The solid lines show the input mixing ratio profiles for different $\mathrm{C} / \mathrm{O}$, with different colors corresponding to different $\mathrm{C} / \mathrm{O}$, as shown by the legend. The retrieved absolute mixing ratios for the different $\mathrm{C} / \mathrm{O}$ are shown with error bars. Note that we retrieve constant-with-altitude mixing ratio profiles. Note also that the vertical position of the retrieved values is arbitrary.

results. Interestingly, the retrieval method using the isothermal approximation was found to bias the results significantly. For example, for $\mathrm{C} / \mathrm{O}=0.7$ the true abundance for $\mathrm{H}_{2} \mathrm{O}$ at 0.1 bars is $2.5 \times 10^{-4}$ and is relatively constant with altitude. The retrieved abundance using the isothermal approximation was found to be (3-4) $\times 10^{-3}$, and $16 \sigma$ away from the true value. On the contrary, the retrieved abundance using the parameterized TP profile is $(1.8-6.3) \times 10^{-4}$ and well within $1 \sigma$ from the true value. For $\mathrm{C} / \mathrm{O}=0.5$ and 0.9 we see similar results: using the TP-PARAM method, the true state is within $1 \sigma-2 \sigma$ of the retrieved values, but if we use the TP-ISO method, the same retrieved values are $15 \sigma$ and $20 \sigma$ away, respectively, from the true state.

The two other molecules that contribute to the spectrum, $\mathrm{CO}$ and $\mathrm{CO}_{2}$, were found to be highly degenerate, but could be retrieved within $1 \sigma-2 \sigma$ using the TP-PARAM method. Using the TP-ISO method, abundances were, however, overestimated. For $\mathrm{CO}_{2}$ we found that, using the parameterized TP profile, the true state is within $2 \sigma$ of the retrieved state for $\mathrm{C} / \mathrm{O}=0.5$ and within $1.5 \sigma$ and $1.1 \sigma$ for $\mathrm{C} / \mathrm{O}=0.7$ and 0.9 , respectively. Using the isothermal profile, we obtain retrieved values that are significantly overestimated and are $11.0 \sigma, 10.2 \sigma$, and $9.6 \sigma$ away from the true state for $\mathrm{C} / \mathrm{O}=0.5,0.7$, and 0.9 , respectively. In these hot oxygen-rich atmospheres the retrieved abundances of $\mathrm{CO}$ and $\mathrm{CO}_{2}$ must, however, be interpreted with caution, as both molecules have the only detectable feature in the same wavelength range $(\approx 4.0-5.5 \mu \mathrm{m})$. From Figure 8 , showing the posterior distribution of $\mathrm{CO}$ and $\mathrm{CO}_{2}$ for $\mathrm{C} / \mathrm{O}=0.7$ using the parameterized TP profile, it can be appreciated that the retrieved absolute abundances for these two molecules are highly degenerate. For $\mathrm{C} / \mathrm{O}<1$ no other molecules could be retrieved, and only upper limits could be obtained.

The transmission spectra of these atmospheres with $\mathrm{C} / \mathrm{O}>1$ show that the dominant molecules are $\mathrm{CH}_{4}, \mathrm{HCN}$, $\mathrm{C}_{2} \mathrm{H}_{2}$, and $\mathrm{CO}$, while all other molecules remain hidden below these stronger absorbers (Figure 4). Only these dominant absorbers could be retrieved, while for all other molecules only 

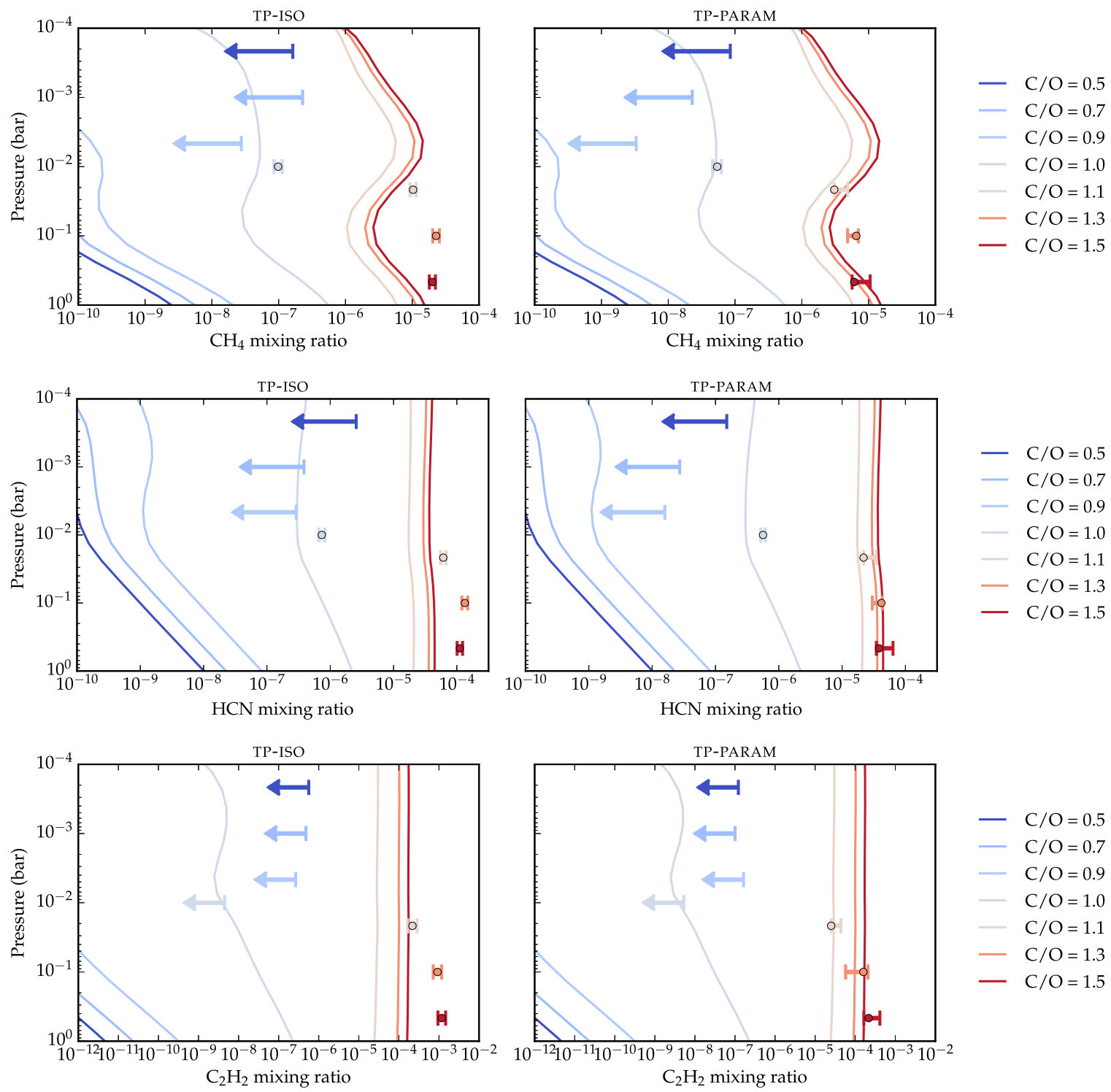

Figure 7. Retrieved $\mathrm{CH}_{4}$ (top), $\mathrm{HCN}$ (middle), and $\mathrm{C}_{2} \mathrm{H}_{2}$ (bottom) abundance for $\mathrm{C} / \mathrm{O}=0.5-1.5$. Caption as in Figure 6 .

upper limits could be placed. We found that also for these carbon-rich atmospheres the TP-PARAM retrieval method gives considerably better results.

Figure 7 shows the retrieved $\mathrm{CH}_{4}, \mathrm{HCN}$, and $\mathrm{C}_{2} \mathrm{H}_{2}$ abundances. For $\mathrm{C} / \mathrm{O}>1$ we can see that the input abundance profiles change significantly as a function of pressure, especially for $\mathrm{CH}_{4}$. In the case of $\mathrm{CH}_{4}$ we found that the TPISO method significantly overestimates the abundances. For all $\mathrm{C} / \mathrm{O}>1$ the retrieved abundances are higher than the true abundances at all pressures in the atmosphere. More reasonable results are obtained with the TP-PARAM method, where the retrieved abundances are always between the maximum and minimum true abundance.

For the same carbon-rich atmospheres, the retrieved abundances of $\mathrm{HCN}$ and $\mathrm{C}_{2} \mathrm{H}_{2}$ using the TP-PARAM approach are all within $1 \sigma-2 \sigma$ of the input abundance, while the values obtained with the TP-ISO are always overestimated by about one order of magnitude and are $8 \sigma-11 \sigma$ away from the true state. Lastly, we note that the retrieved abundances of $\mathrm{CO}$ were within $1 \sigma$ of the true state using the TP-PARAM method, while the same values are an order of magnitude higher than the true state and have underestimated error bars when using the TP-ISO method.

This case with $\mathrm{C} / \mathrm{O}=1$ is the most peculiar as many molecules are visible in the spectrum, and their abundance varies significantly as a function of altitude. In the case of $\mathrm{H}_{2} \mathrm{O}$, $\mathrm{CO}_{2}$, and $\mathrm{CH}_{4}$ the true abundance profile changes by about one order of magnitude at the typical pressures probed by transmission spectra $\left(10^{-1}-10^{-4}\right.$ bars; see Figure 3$)$. For $\mathrm{H}_{2} \mathrm{O}$, small differences are seen between the TP-PARAM and TPISO methods. The retrieved abundances are (1.4-1.6) $\times 10^{-6}$ in the first case and (1.6-1.9) $\times 10^{-6}$ in the second case, while the input profile varies between $5 \times 10^{-7}$ and $4 \times 10^{-6}$ for pressures between 1 and $10^{-4}$ bars. For carbon monoxide, in both cases the retrieved abundances are overestimated by about one order of magnitude, with values $6 \sigma-7.5 \sigma$ away from the true state. This is somewhat surprising, considering that the input profile is constant with altitude. For $\mathrm{CO}_{2}$ the retrieved abundance is within $1 \sigma$ using the TP-PARAM method and within $2 \sigma$ using the TP-ISO approach. Finally, for $\mathrm{CH}_{4}$ and $\mathrm{HCN}$ the retrieved abundances are very similar using both methods and are found to be within the maximum and minimum abundances 


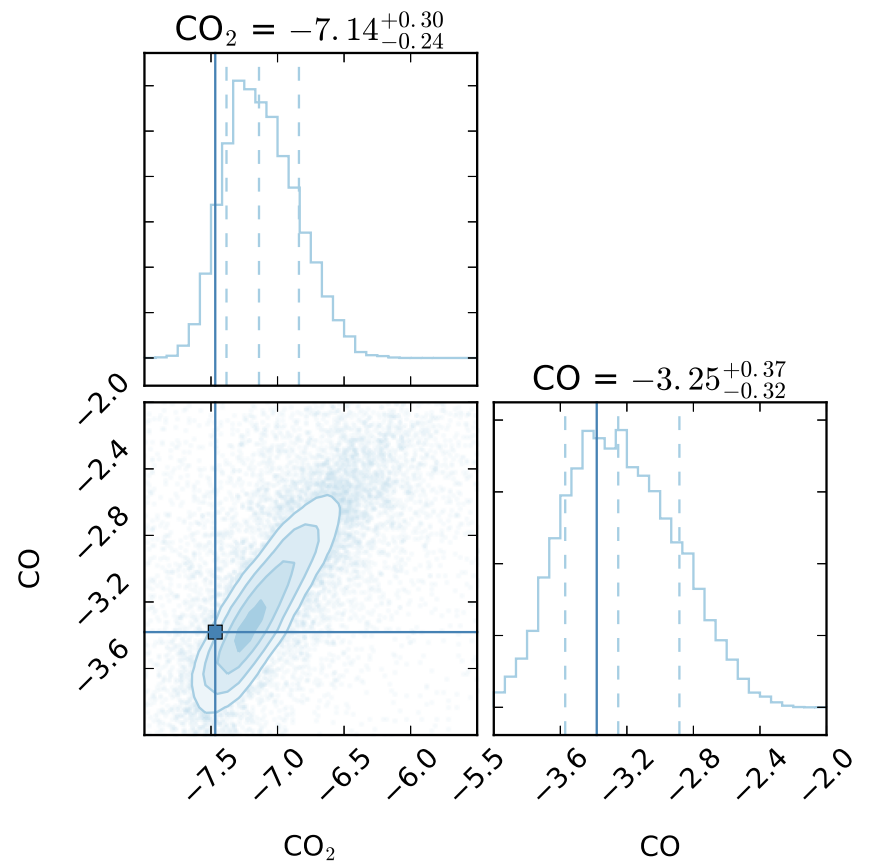

Figure 8. Posterior distributions of $\mathrm{CO}$ and $\mathrm{CO}_{2}$ for $\mathrm{C} / \mathrm{O}=0.7$ for the retrieval approach with a parameterized TP profile. Dashed lines in the histogram plots show the $1 \sigma$ confidence intervals. The true state (absolute input abundance at 0.1 bars) is shown with a blue square box and straight blue lines. Note that the mixing ratios of these two molecules are approximately constant with altitude in this case, as seen in Figure 3.

of the input profiles, which both vary significantly as a function of altitude.

\section{DISCUSSION}

\subsection{The Impact of Common Approximations}

The results presented in the previous section highlight how common assumptions used in current retrieval methods for exoplanets can potentially lead to wrong conclusions.

Strong biases are seen for all $\mathrm{C} / \mathrm{O}$ ratios, where we see that the isothermal approximation causes in general an overestimation of the absolute abundances by one order of magnitude and significantly underestimates error bars.

The strongest biases are seen for $\mathrm{H}_{2} \mathrm{O}, \mathrm{CO}$, and $\mathrm{CO}_{2}$ in the $\mathrm{C} / \mathrm{O}<1$ atmospheres, and for $\mathrm{HCN}, \mathrm{CH}_{4}$, and $\mathrm{C}_{2} \mathrm{H}_{2}$ in the $\mathrm{C} / \mathrm{O}>1$ atmospheres. This is not surprising, given that these are the strongest absorbers for these $\mathrm{C} / \mathrm{O}$ ranges, and therefore those with the smallest retrieved uncertainties.

For all these atmospheres, excluding $\mathrm{C} / \mathrm{O}=1$, the retrieval method assuming a parameterized $\mathrm{TP}$ profile was found to describe the more complex temperature structure of the atmosphere, leading to retrieved values in general agreement with the true state within $1 \sigma$ on average. This finding opens even new prospects for the use of this technique to characterize exoplanetary atmospheres, showing how high signal-to-noise ratio and broad wavelength coverage transmission spectra can lead to significant constraints on the temperature profiles of the terminator region of hot Jupiter atmospheres.

In general, the retrieval of constant-with-altitude mixing ratio profiles seems sufficient to describe the more complex real profiles when the TP-PARAM approach is used, and it is therefore a fair approximation in most cases. This is especially true for the $\mathrm{C} / \mathrm{O}<1$ atmospheres, where the true profiles of the most abundant molecules are constant, but it is also true for the $\mathrm{C} / \mathrm{O}>1$ atmospheres, where one of the most abundance molecules, $\mathrm{CH}_{4}$, has a profile that varies significantly with altitude. The retrieved abundance of this molecule falls within the minimum and maximum true abundance, indicating that the features seen in the transmission spectra at 3.4 and $7.6 \mu \mathrm{m}$ probe similar pressure regions in the atmosphere.

Retrieved parameters are more strongly affected for the $\mathrm{C} / \mathrm{O}=1$ case, where the biases introduced by assuming a constant-with-altitude abundance profile dominate. Small differences in the retrieved values are seen using the TPPARAM and TP-ISO methods, and the retrieved results are in both cases several sigma away from the true state. Interestingly, the TP profile retrieved using the TP-PARAM method is also several sigma away from the input profile. This indicates that the biases are driven by the assumption that the abundance profiles are constant with altitude, which is clearly wrong for most molecules. In this case, the different features of the same molecules seen at different wavelengths (e.g., $\mathrm{H}_{2} \mathrm{O}$ and $\mathrm{CO}$ ) probe different regions of the atmosphere, where the abundances can vary significantly. Trying to fit these features using the same abundances throughout the entire atmosphere clearly leads to strong biases. We did not explore here the possibility of fitting a more complex abundance profile for the molecules, but future work in this direction will be required.

The retrieved abundances obtained with the TP-PARAM method will enable placing some limits on the $\mathrm{C} / \mathrm{O}$ values of the observed atmospheres. First, it will be clearly possible to differentiate between $\mathrm{C} / \mathrm{O}$ greater or less than unity and $\mathrm{C} / \mathrm{O}=1$, as the spectrum signatures change dramatically at this threshold. Tighter constraints on $\mathrm{C} / \mathrm{O}$ can be obtained by linking the retrieved absolute abundances with atmospheric chemical models. However, our results indicate that it will be difficult. For $\mathrm{C} / \mathrm{O}<1$, the strongest tracer for $\mathrm{C} / \mathrm{O}$ is water. Increasingly lower $\mathrm{H}_{2} \mathrm{O}$ abundances are expected at increasing $\mathrm{C} / \mathrm{O}$, but the differences seen here are rather small and comparable with the retrieved uncertainties (see Figure 6). Similarly, for $\mathrm{C} / \mathrm{O}>1$, the strongest tracers are $\mathrm{HCN}$ and $\mathrm{C}_{2} \mathrm{H}_{2}$ (and, to a lesser extent, $\mathrm{CH}_{4}$, which has, however, a nonuniform abundance profile). However, even in this case the difference in absolute abundance is quite small and comparable with the error bars of the retrieved values. This is not totally surprising, given that the simulated transmission spectra show very little variation between similar $\mathrm{C} / \mathrm{O}$ in both the oxygenand carbon-rich regimes. Higher signal-to-noise ratio observations might further decrease these uncertainties and therefore improve the inferred $\mathrm{C} / \mathrm{O}$, but we note that we are already very close to the systematic uncertainties. Other techniques might prove more effective at constraining the $\mathrm{C} / \mathrm{O}$ ratio, such as emission spectra through secondary eclipse measurements and/ or using chemically consistent retrieval approaches (see, e.g., Greene et al. 2016).

\subsection{Understanding the Biases}

In order to understand why, and in which scenarios, a nonisothermal profile and constant-with-altitude abundance profiles might lead to strong biases, it is instructive to look at the spectral transmittance as a function of pressure for the atmospheres under study. Figure 9 shows the spectral transmittance integrated over the path parallel to the line of sight as a function of pressure, together with the temperature and scale height profiles. It can be seen that different spectral 

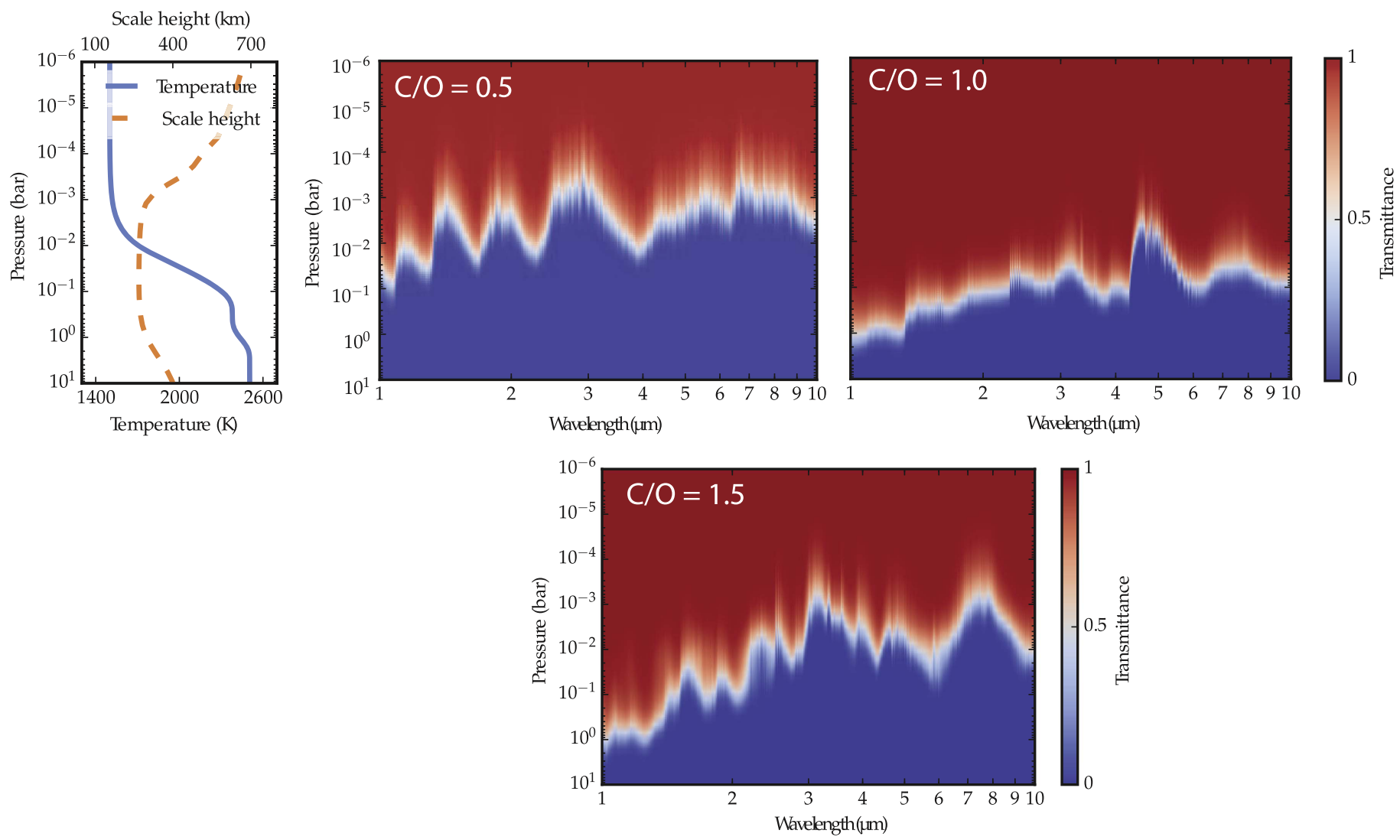

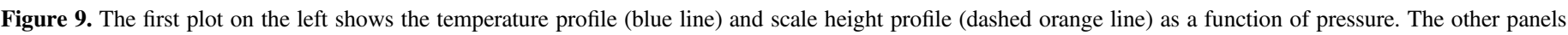

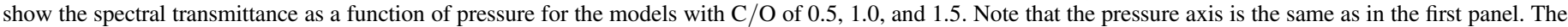

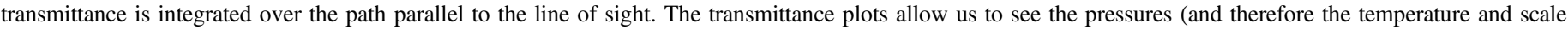
height) probed at different wavelengths for different $\mathrm{C} / \mathrm{O}$ regimes.

regions probe different pressure ranges and therefore different temperatures and scale heights. First, we note that the scale height does not increase exponentially with altitude between $10^{-3}$ and $1 \mathrm{bar}$, as one would expect in a purely isothermal atmosphere. On the contrary, the strong temperature gradient seen at these pressures causes the scale height to stay relatively constant at $\approx 200 \mathrm{~km}$. For the atmosphere with $\mathrm{C} / \mathrm{O}=0.5$ we see that most of the absorption occurs between $10^{-4}$ and $10^{-1}$ bar, while for the $\mathrm{C} / \mathrm{O}=1.1$ case the transmission spectrum probes higher-pressure regions, from $1 \mathrm{bar}$ to $10^{-3}$ bars. At these pressures the temperature varies from 1500 to about $2500 \mathrm{~K}$. We also note that the peak of the absorption features probes the higher-altitude and lowertemperature part of the atmospheres, while the troughs probe the regions of the atmosphere that are almost $1000 \mathrm{~K}$ hotter.

An isothermal approximation will clearly lead to several problems. First, as we noted before, the scale height of an isothermal atmosphere will increase exponentially, while in this case it is roughly constant with pressure up to $1 \mathrm{mbar}$. Spectral features that probe different pressures, such as the strong water features seen for $\mathrm{C} / \mathrm{O}<1$, will therefore vary considerably if the scale height is constant with pressure or not. A second, equally important effect is caused by the very different temperatures probed. Molecular opacity cross sections vary considerably between the temperature regions probed here (1500-2500 K), and therefore assuming a single temperature will obviously lead to further biases.

Additionally, Figure 9 helps to explain why for the retrievals of the atmospheres with $\mathrm{C} / \mathrm{O}<1$ we found that the fit of the low-altitude temperature improves for higher $\mathrm{C} / \mathrm{O}$, while that for the high-altitude part of the TP profile improves for lower $\mathrm{C} / \mathrm{O}$. As the $\mathrm{C} / \mathrm{O}$ value increases from 0.5 to 0.9 , we see that the water abundance decreases from about $4 \times 10^{-4}$ to $1 \times 10^{-4}$. The effect in the transmission spectrum is a vertical shift toward lower absorption, which also translates into a vertical shift in the transmissivity plot. This means that as the water content drops, we probe increasingly higher pressure regions of the atmospheres, meaning that we increasingly lose information from the upper-altitude part of the atmosphere. This easily explains why the uncertainty on the retrieved upperaltitude temperature of these atmospheres progressively increases, while the constraint of the temperature in the bottom layers improves for higher $\mathrm{C} / \mathrm{O}$.

So far we have only considered cloud-free, broad wavelength range observations. This is the case where common approximations are most likely to break down. Shorter wavelength ranges will, for example, tend to probe specific regions of the TP profiles. For instance, an atmosphere with $\mathrm{C} / \mathrm{O}=1.1$ observed between 1 and $3 \mu \mathrm{m}$ will only probe pressures between 1 and 0.1 bars, where the temperature is roughly constant at $\approx 2400 \mathrm{~K}$. In this scenario, we expect the isothermal approximation to be sufficiently good. However, this is not always the case. If the atmosphere with $\mathrm{C} / \mathrm{O}=0.5$ is observed between 2.5 and $4 \mu \mathrm{m}$, we will see a strong water feature with a peak absorption coming from a region with a temperature of about $1500 \mathrm{~K}$, and with wings probing increasingly higher temperatures. Clearly, even in this case an isothermal approximation would give biased results, and our 

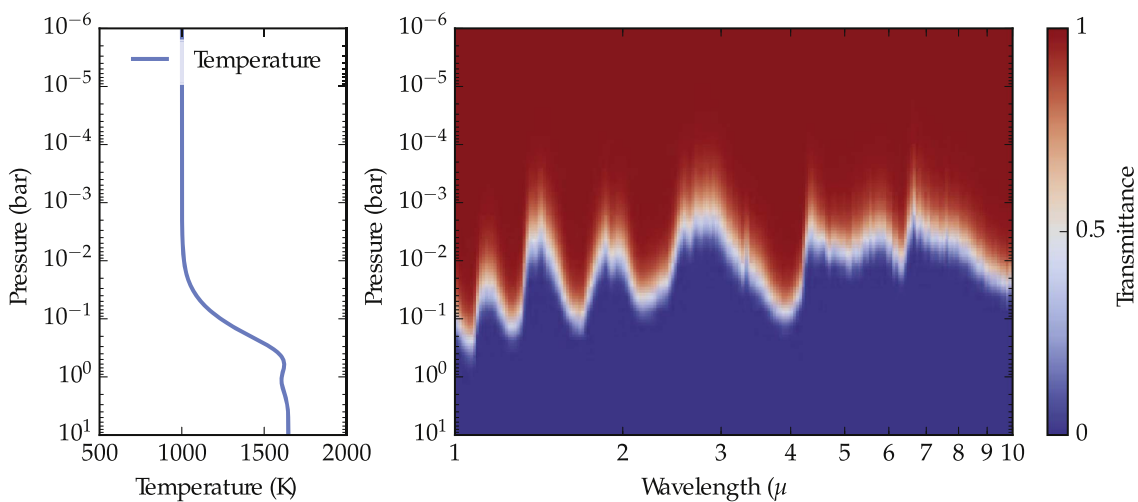

Figure 10. TP profile and spectral transmittance for a planet with a cooler TP profile. Caption as in Figure 9.

study indicates that the retrieved uncertainty of the abundance will be likely underestimated.

The presence of uniform clouds will increase the degeneracy of model parameters, somewhat hiding the underlying biases, as the effect of a cloud deck is that of making the atmosphere opaque. A cloud deck extending to 10 mbars would, for example, make the atmosphere opaque to incoming radiation for pressures higher than 10 mbars. This also means that it will be impossible to probe the temperature and mixing ratio profiles in this pressure regime. In the case under study, the TP profile for pressure lower than 10 mbars is relatively isothermal, and in the presence of clouds, an isothermal approximation would therefore be appropriate. Note, however, that cloud models commonly used in current retrievals were found to cause significant degeneracies. Line \& Parmentier (2016) investigated the biases of retrieving a uniform cloud cover in the presence of patchy clouds and found significant degeneracies in the retrieved mean molecular weight.

We also note that similar biases are expected for cooler planets. Figure 10 shows the spectral transmittance as a function of pressure for an atmosphere with a cooler TP profile, with high-altitude temperature of $1000 \mathrm{~K}$. The spectrum was computed from a chemical model with $\mathrm{C} / \mathrm{O}=0.5$ and assuming the same hot Jupiter used in this work. It can be seen that the spectrum probes the range of pressures $\left(10^{-3}\right.$ 1 bar) where the TP profile changes more significantly. We therefore expect that the use of an isothermal profile to retrieve this spectrum will lead to similar biases to those found for the hotter planet case.

Lastly, we note that this study focused on two specific common assumptions in current retrieval methods, constant mixing ratio and temperature profiles, and the biases that these approximations can lead to. However, other strong assumptions are likely to bias our retrievals. One of the most important ones is to neglect 3D dynamical effects. The simulated observations have in fact been generated using 1D chemical models and assume a uniform chemistry and atmospheric temperature at the terminator region. Further studies that compare transmission spectra obtained with general circulation models and retrieved with the simpler 1D models are needed to address the biases of this assumption. A recent study in this direction is presented in Feng et al. (2016).

\section{CONCLUSIONS}

In this paper we investigated the biases caused by two common assumptions in the forward models used by current retrieval methods of transmission spectra of hot Jupiter atmospheres: the use of an isothermal profile and constantwith-altitude abundances. We investigated whether these assumptions will still be valid for high signal-to-noise ratio, broad wavelength coverage spectra such as those expected by $J W S T$. In order to do this, we simulated high-quality observations using a chemical scheme developed by Venot et al. (2012), which include detailed temperature and abundance profiles, and we retrieved them using two simpler forward models: the first one assumes an isothermal profile (TPISO), while the second one assumes a parameterized temperature profile (TP-PARAM). In both cases, constant-with-altitude abundances were retrieved. We found the following:

1. The nonuniform temperature profile could be well retrieved within about $1 \sigma$ for all cases but $\mathrm{C} / \mathrm{O}=1$ using the TP-PARAM method. This is an important result, opening the possibility to obtain detailed temperature structure information about the terminator region of a hot Jupiter.

2. The retrieval approach that assumes an isothermal profile led to strong biases. We found that, on average, the retrieved abundances using this method are overestimated by about one order of magnitude and the error bars are underestimated. The TP-PARAM approach leads to much improved constraints, with retrieved abundances within $1 \sigma-2 \sigma$ of the input values in most cases. This is also because the retrieved uncertainties are generally larger.

3. The retrieval assumption that abundance profiles are constant with altitude was found to be a good approximation for $\mathrm{C} / \mathrm{O}<1$ and $\mathrm{C} / \mathrm{O}>1$ atmospheres, but not for $\mathrm{C} / \mathrm{O}=1$. In the latter case, most of the abundance profiles have strong variations, and a uniform abundance profile is a poor approximation that leads to significant biases. Future work will therefore be needed to address the feasibility of fitting more complex abundance profiles.

4. Although we found that differentiating between $\mathrm{C} / \mathrm{O}<1, \mathrm{C} / \mathrm{O}=1$, and $\mathrm{C} / \mathrm{O}>1$ was straightforward, we also found that tighter constraints are more difficult to obtain as the differences between the transmission spectra are relatively small. Higher signal-to-noise ratio observations might lead to better constraints, but other biases, due to systematic uncertainties, for example, might become more dominant. Emission spectra observations, possibly combined with transmission spectra, might give better constraints than transmission spectra alone. 
These results show that when broad wavelength ranges and high signal-to-noise ratio observations are used, the forward models used in our retrieval approaches need to allow for larger flexibility. One very simple solution is to adopt a parameterization of the TP profile, as the one used here, but other techniques, such as the two-stage approach used in Waldmann et al. (2015a), could be considered in the future.

We thank the referee for providing useful comments. This work was supported by STFC (ST/K502406/1) and the ERC projects ExoLights (617119) and ExoMol (267219). O.V. acknowledges support from the KU Leuven IDO project IDO/ 10/2013 and from the FWO Postdoctoral Fellowship program. P.O.L. acknowledges support from the LabEx P2IO, the French ANR contract 05-BLAN-NT09-573739.

\section{APPENDIX}

\section{REFERENCES}

Agúndez, M., Venot, O., Selsis, F., \& Iro, N. 2014, ApJ, 781, 68

Ali-Dib, M., Mousis, O., Petit, J.-M., \& Lunine, J. I. 2014, ApJ, 785, 125

Anderlohr, J. M., Pires da Cruz, A., Bounaceur, R., \& Battin-Leclerc, F. 2010, CST, 182, 39

Barber, R. J., Strange, J. K., Hill, C., et al. 2014, MNRAS, 437, 1828

Barber, R. J., Tennyson, J., Harris, G. J., \& Tolchenov, R. N. 2006, MNRAS, 368, 1087

Barstow, J. K., Aigrain, S., Irwin, P. G. J., et al. 2013, MNRAS, 430, 1188

Barstow, J. K., Aigrain, S., Irwin, P. G. J., Kendrew, S., \& Fletcher, L. N. 2015, MNRAS, 448, 2546

Barstow, J. K., \& Irwin, P. G. J. 2016, MNRAS, 461, L92

Batalha, N., Kalirai, J., Lunine, J., Clampin, M., \& Lindler, D. 2015, arXiv: 1507.02655

Battin-Leclerc, F., Bounaceur, R., Glaude, P.-A., \& Belmekki, N. 2006, arXiv: physics $/ 0603102$

Bean, J. L., Desert, J.-M., Seifahrt, A., et al. 2013, ApJ, 771, 108

Beaulieu, J. P., Kipping, D., Batista, V., et al. 2010, MNRAS, 409, 963

Beichman, C., Benneke, B., Knutson, H. A., et al. 2014, PASP, 126, 1134

Benneke, B., \& Seager, S. 2012, ApJL, 753, 100

Benneke, B., \& Seager, S. 2013, ApJ, 778, 153

Bounaceur, R., Glaude, P. A., Fournet, R., et al. 2007, Int. J. Veh. Des., 44, 1

Brogi, M., de Kok, R. J., Birkby, J. L., Schwarz, H., \& Snellen, I. A. G. 2014, A\&A, 565, A124

Brown, T. M. 2001, ApJL, 553, 1006

Cavalié, T., Moreno, R., Lellouch, E., et al. 2014, A\&A, 562, A33

Charbonneau, D., Brown, T. M., Noyes, R. W., \& Gilliland, R. L. 2002, ApJL, 568,377

Charbonneau, D., Knutson, H. A., Barman, T. S., et al. 2008, ApJ, 686, 1341

Cowan, N. B., Greene, T., Angerhausen, D., et al. 2015, PASP, 127, 311

Danielski, C., Deroo, P., Waldmann, I., et al. 2014, ApJ, 785, 35

Deming, D., Knutson, H. A., Agol, E., et al. 2011, ApJ, 726, 95

de Wit, J., \& Seager, S. 2013, Sci, 342, 1473

Doyon, R., Hutchings, J. B., Beaulieu, M., et al. 2012, Proc. SPIE, 8442 , $84422 \mathrm{R}$

Feng, Y. K., Line, M. R., Fortney, J., et al. 2016, ApJ, 829, 52

Feroz, F., \& Hobson, M. P. 2008, MNRAS, 384, 449

Ferruit, P., Birkmann, S., Böker, T., et al. 2014, Proc. SPIE, 9143, 91430A

Greene, T., Beichman, C., Eisenstein, D., et al. 2007, Proc. SPIE, 6693, $66930 \mathrm{G}$

Greene, T. P., Line, M. R., Montero, C., et al. 2016, ApJ, 817, 17

Grillmair, C. J., Charbonneau, D., Burrows, A., et al. 2007, ApJL, 658, L115

Guillot, T. 2010, A\&A, 520, A27

Harris, G. J., Tennyson, J., Kaminsky, B. M., Pavlenko, Y. V., \& Jones, H. R. A. 2006, MNRAS, 367, 400
Hollis, M. D. J., Tessenyi, M., \& Tinetti, G. 2013, CoPhC, 184, 2351 Husser, T. O., Wende-von Berg, S., Dreizler, S., et al. 2013, A\&A, 553, 6 Irwin, P. G. J., Teanby, N. A., De Kok, R., et al. 2008, JQSRT, 109, 1136 Kendrew, S., Scheithauer, S., Bouchet, P., et al. 2015, PASP, 127, 623

Knutson, H. A., Charbonneau, D., Allen, L. E., et al. 2007, Natur, 447, 183 Knutson, H. A., Madhusudhan, N., Cowan, N. B., et al. 2011, ApJ, 735, 27

Kreidberg, L., Bean, J. L., Desert, J.-M., et al. 2014, Natur, 505, 69

Lee, J. M., Fletcher, L. N., \& Irwin, P. G. J. 2011, MNRAS, 420, 170

Lewis, N. K., Showman, A. P., Fortney, J., et al. 2010, ApJ, 720, 344

Line, M. R., Fortney, J., Marley, M. S., \& Sorahana, S. 2014, ApJ, 793, 33

Line, M. R., \& Parmentier, V. 2016, ApJ, 820, 78

Line, M. R., Vasisht, G., Chen, P., Angerhausen, D., \& Yung, Y. L. 2011, ApJ, 738, 32

Line, M. R., Wolf, A. S., Zhang, X., et al. 2013, ApJ, 775, 137

Line, M. R., Zhang, X., Vasisht, G., et al. 2012, ApJ, 749, 93

Liou, K. N. 2002, An Introduction to Atmospheric Radiation (New York: Academic Press)

Madhusudhan, N. 2012, ApJ, 758, 36

Madhusudhan, N., Burrows, A., \& Currie, T. 2011a, ApJ, 737, 34

Madhusudhan, N., Harrington, J., Stevenson, K. B., et al. 2011b, Natur, 469, 64

Madhusudhan, N., \& Seager, S. 2009, ApJ, 707, 24

Moses, J. I., Line, M. R., Visscher, C., et al. 2013a, ApJ, 777, 34

Moses, J. I., Madhusudhan, N., Visscher, C., \& Freedman, R. S. 2013b, ApJ, 763,25

Moses, J. I., Visscher, C., Fortney, J., et al. 2011, ApJL, 737, 15

Mousis, O., Fletcher, L. N., Lebreton, J. P., et al. 2014, P\&SS, 104, 29

Öberg, K. I., Murray-Clay, R., \& Bergin, E. A. 2011, ApJL, 743, L16

Parmentier, V., \& Guillot, T. 2014, A\&A, 562, A133

Parmentier, V., Guillot, T., Fortney, J., \& Marley, M. S. 2015, A\&A, 574, A35

Parmentier, V., Showman, A. P., \& Lian, Y. 2013, A\&A, 558, A91

Redfield, S., Endl, M., Cochran, W. D., \& Koesterke, L. 2008, ApJL, 673, L87

Richard, C., Gordon, I. E., Rothman, L. S., et al. 2012, JQSRT, 113, 1276

Rothman, L. S., Gordon, I. E., Babikov, Y., et al. 2013, JQSRT, 130, 4

Rothman, L. S., Gordon, I. E., Barber, R. J., et al. 2010, JQSRT, 111, 2139

Seager, S. 2011, ConPh, 52, 602

Sing, D. K., Fortney, J., Nikolov, N., et al. 2016, Natur, 529, 59

Snellen, I. A. G., Albrecht, S., de Mooij, E. J. W., \& Le Poole, R. S. 2008 A\&A, 487, 357

Stevenson, K. B., Harrington, J., Nymeyer, S., et al. 2010, Natur, 464, 1161

Swain, M., Deroo, P., Griffith, C. A., et al. 2010, Natur, 463, 637

Swain, M., Deroo, P., Tinetti, G., et al. 2013, Icar, 225, 432

Tennyson, J., \& Yurchenko, S. N. 2012, MNRAS, 425, 21

Thiabaud, A., Marboeuf, U., Alibert, Y., Leya, I., \& Mezger, K. 2015, A\&A, 580, A30

Tinetti, G., Deroo, P., Swain, M., et al. 2010, ApJL, 712, L139

Tinetti, G., Tennyson, J., Griffith, C. A., \& Waldmann, I. 2012, RSPTA, 370,2749

Tinetti, G., Vidal-Madjar, A., Liang, M.-C., et al. 2007, Natur, 448, 169

Todorov, K. O., Deming, D., Knutson, H. A., et al. 2013, ApJ, 770, 102

Tsiaras, A., Rocchetto, M., Waldmann, I., et al. 2016, ApJ, 820, 99

Tsiaras, A., Waldmann, I., Rocchetto, M., et al. 2015, arXiv:1511.07796

Valencia, D., Guillot, T., Parmentier, V., \& Freedman, R. S. 2013, ApJ, 775,10

Venot, O., \& Agúndez, M. 2015, ExA, 40, 469

Venot, O., Agúndez, M., Selsis, F., Tessenyi, M., \& Iro, N. 2014, A\&A, 562,51

Venot, O., Fray, N., Bénilan, Y., et al. 2013, A\&A, 551, A131

Venot, O., Hébrard, E., Agúndez, M., et al. 2012, A\&A, 546, A43

Venot, O., Hébrard, E., Agúndez, M., Decin, L., \& Bounaceur, R. 2015, A\&A, 577, A33

Waldmann, I., Rocchetto, M., Tinetti, G., et al. 2015b, ApJ, 813, 13

Waldmann, I., Tinetti, G., Drossart, P., et al. 2012, ApJL, 744, 35

Waldmann, I., Tinetti, G., Rocchetto, M., et al. 2015a, ApJ, 802, 107

Wang, H., Warner, S. J., Oehlschlaeger, M. A., et al. 2010, CoFl, 157, 1976

Yurchenko, S. N., Barber, R. J., \& Tennyson, J. 2011, MNRAS, 413, 1828

Yurchenko, S. N., Tennyson, J., Barber, R. J., \& Thiel, W. 2013, JMoSp, 291, 69

Zellem, R. T., Griffith, C. A., Deroo, P., Swain, M., \& Waldmann, I. 2014, ApJ, 796, 48 